Article

\title{
Genetic Diversity of Potassium Ion Channel Proteins Encoded by Chloroviruses That Infect Chlorella heliozoae
}

\author{
Carter R. Murry ${ }^{1,2}$, Irina V. Agarkova ${ }^{2,3}$, Jayadri S. Ghosh ${ }^{2,3}$, Fiona C. Fitzgerald ${ }^{4}$, \\ Roger M. Carlson ${ }^{2}$, Brigitte Hertel ${ }^{5}$, Kerri Kukovetz ${ }^{5}$, Oliver Rauh ${ }^{5}$, Gerhard Thiel ${ }^{5}$ and \\ James L. Van Etten 2,3,*(D) \\ 1 School of Biological Sciences-Microbiology Program, University of Nebraska-Lincoln, Lincoln, \\ NE 68588-0118, USA; murry.carter12@gmail.com \\ 2 Nebraska Center for Virology, University of Nebraska-Lincoln, Lincoln, NE 68583-0900, USA; \\ irina@unl.edu (I.V.A.); jghosh2@unl.edu (J.S.G.); roger.carlson@unl.edu (R.M.C.) \\ 3 Department of Plant Pathology, University of Nebraska-Lincoln, Lincoln, NE 68583-0833, USA \\ 4 Department of Chemistry and Biochemistry, Benedictine College, Atchison, KS 66002, USA; \\ f.fitzgerald559@gmail.com \\ 5 Membrane Biophysics, Department of Biology, Technische Universitat, 64287 Darmstadt, Germany; \\ hertelb@bio.tu-darmstadt.de (B.H.); kukovetz@bio.tu-darmstadt.de (K.K.); rauh@bio.tu-darmstadt.de (O.R.); \\ thiel@bio.tu-darmstadt.de (G.T.) \\ * Correspondence: jvanetten1@unl.edu; Tel.: +1-402-472-3168
}

Received: 3 June 2020; Accepted: 19 June 2020; Published: 23 June 2020

\begin{abstract}
Chloroviruses are large, plaque-forming, dsDNA viruses that infect chlorella-like green algae that live in a symbiotic relationship with protists. Chloroviruses have genomes from 290 to $370 \mathrm{~kb}$, and they encode as many as 400 proteins. One interesting feature of chloroviruses is that they encode a potassium ion $\left(\mathrm{K}^{+}\right)$channel protein named $\mathrm{Kcv}$. The $\mathrm{Kcv}$ protein encoded by SAG chlorovirus ATCV-1 is one of the smallest known functional $\mathrm{K}^{+}$channel proteins consisting of 82 amino acids. The $\mathrm{Kcv}_{\text {ATCV-1 }}$ protein has similarities to the family of two transmembrane domain $\mathrm{K}^{+}$channel proteins; it consists of two transmembrane $\alpha$-helixes with a pore region in the middle, making it an ideal model for studying $\mathrm{K}^{+}$channels. To assess their genetic diversity, $k c v$ genes were sequenced from 103 geographically distinct SAG chlorovirus isolates. Of the $103 \mathrm{kcv}$ genes, there were 42 unique DNA sequences that translated into 26 new Kcv channels. The new predicted Kcv proteins differed from $\mathrm{Kcv}_{\mathrm{ATCV}-1}$ by 1 to 55 amino acids. The most conserved region of the Kcv protein was the filter, the turret and the pore helix were fairly well conserved, and the outer and the inner transmembrane domains of the protein were the most variable. Two of the new predicted channels were shown to be functional $\mathrm{K}^{+}$channels.
\end{abstract}

Keywords: Chloroviruses; potassium ion channels; Kcv channels; algal viruses

\section{Introduction}

Chloroviruses (family Phycodnaviridae) are large, plaque-forming, dsDNA viruses that infect certain chlorella-like green algae that live in a symbiotic relationship with protists [1]. Chloroviruses have an internal membrane and they are icosahedral in shape with a spike structure at one of their vertices [2]. They have genomes that are 290 to $370 \mathrm{~kb}$ in size and are predicted to encode up to 400 proteins (CDSs) and 16 tRNAs. These viruses are ubiquitous in nature and have been isolated from freshwater ponds, lakes, and rivers across the globe. There are four groups of chloroviruses based on the host they infect: viruses that infect Chlorella variabilis NC64A (referred to as NC64A viruses), 
viruses that infect Chlorella variabilis Syngen 2-3 (referred to as Osy viruses), viruses that infect Chlorella heliozoae SAG 3.83 (referred to as SAG viruses), and viruses that infect Micratinium conductrix Pbi, (referred to as Pbi viruses). The most studied chlorovirus is the NC64A virus Paramecium bursaria chlorella virus 1 (PBCV-1); its host, C. variabilis NC64A, lives in symbiosis with Paramecium bursaria.

The PBCV-1 genome is $\sim 331 \mathrm{~kb}$ and encodes 416 predicted CDSs and $11 \mathrm{tRNA}$ genes. About half of the identified CDSs resemble proteins of known function, including some that are novel for a virus. One protein that PBCV-1, as well as most of the chloroviruses, encodes is a potassium ion $\left(\mathrm{K}^{+}\right)$channel protein (named Kcv) [3]. When the 94 amino acid $\mathrm{KcV}_{\mathrm{PBCV}}-1$ was discovered, it was the smallest protein known to form a functional $\mathrm{K}^{+}$channel. The $\mathrm{KCV}_{\mathrm{PBCV}}-1$ protein consists of only the basic functional units that are present in all $\mathrm{K}^{+}$channels in that it has a short slide helix, an outer transmembrane helix, a turret, a pore helix, a filter, and an inner transmembrane helix (Figure 1); four of these proteins form a functional $\mathrm{K}^{+}$channel.

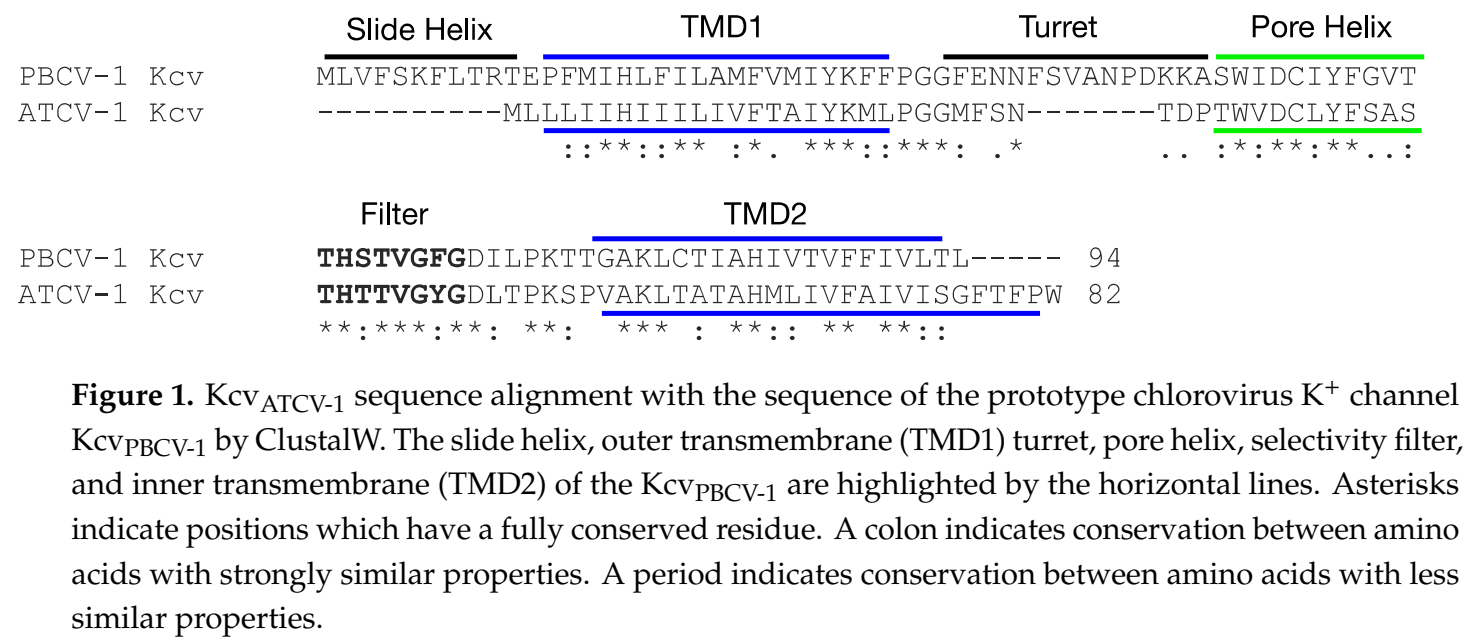

$\mathrm{K}_{\mathrm{PBCV}-1}$ is hypothesized to play an important role during infection of its host. After the virus attaches to the host cell wall and degrades the wall at the point of attachment, the PBCV-1 internal membrane fuses with the host's plasma membrane [4]. The Kcv channel is located in the virus's internal membrane [5], and once the two membranes are fused, the Kcv channel becomes part of the host membrane. This allows Kcv to participate in the rapid depolarization of the host cell membrane [6,7] and the release of $\mathrm{K}^{+}$from the cell [8].

The rapid loss of $\mathrm{K}^{+}$from the host and associated water fluxes significantly reduce the host turgor pressure, which aids ejection of viral DNA and virion-associated proteins into the host [9]. Host membrane depolarization also inhibits many host secondary transporters [10] and prevents infection by a second virus [11]. Because of the small size of Kcv, it has served as an excellent model for studying $\mathrm{K}^{+}$channels and there are over 60 research publications on $\mathrm{Kcv}$ channels.

Since the discovery of $\mathrm{KCV}_{\mathrm{PBCV}-1}$, even smaller chlorovirus-encoded $\mathrm{K}^{+}$channel proteins have been described including an 82 amino acid protein from SAG chlorovirus, ATCV-1, referred to as $\mathrm{KcV}_{\text {ATCV-1 }}$ (Figure 1). Expression studies established that $\mathrm{KCv}_{\text {ATCV-1 }}$ makes a functional, $\mathrm{K}^{+}$selective channel in Xenopus laevis oocytes and in yeast [12]. The objective of this study was to isolate and analyze the sequence diversity of the kcv gene from 103 SAG chloroviruses that come from freshwater collected throughout the world. Ultimately, one can anticipate some physiological differences among the Kcv channels from the SAG viruses.

\section{Materials and Methods}

\subsection{Cultures}

Water samples were collected from lakes, ponds, and rivers from around the United States, Canada, Guatemala, Brazil, Chile, Germany, and Greenland (Table A1 (Appendix A)). The samples were passed 
through a $0.45 \mu \mathrm{m}$ filter (PES filters, Sartorius, Gottingen, Germany). Chloroviruses were isolated from the filtered water samples using a plaque assay on a C. heliozoae SAG 3.83 lawn. For the plaque assay, plates were made using Modified Bold's Basal Medium (MBBM) 1.5\% agar with tetracycline added at $10 \mu \mathrm{g} / \mathrm{mL}$ [13]. Each plate was filled with about $20 \mathrm{~mL}$ of MBBM agar and allowed to solidify. In a tube, $2.5 \mathrm{~mL}$ of $0.75 \% \mathrm{MBBM}$ agar was combined with $1 \mathrm{~mL}$ of the filtered water sample and $300 \mu \mathrm{L}$ of C. heliozoae cells $\left(\sim 1.5 \times 10^{8}\right.$ cells $\left./ \mathrm{mL}\right)$. The tube was mixed and poured over the solidified $1.5 \% \mathrm{MBBM}$ agar plate. Once the top agar layer solidified, the plates were inverted and kept under constant light at $25{ }^{\circ} \mathrm{C}$ for a few days. If SAG chloroviruses were present in the water sample, plaques formed on the plates. Two to three unique plaques (e.g., different size and sometimes different shape plaques) were picked from each plate with a sterile toothpick and placed in a $1.5 \mathrm{~mL}$ tube filled with C. heliozoae cells. These samples were then placed on a spinning wheel for 1 day to propagate the virus. The resulting lysates were serially diluted to $10^{-6}$ in virus suspension buffer (VSB, $50 \mathrm{mM}$ Tris $\mathrm{HCl}, 10 \mathrm{mM} \mathrm{MgCl}$, $\mathrm{pH} 7.8$ ) and $100 \mu \mathrm{L}$ of the resultant dilution was plaqued. Each virus sample was plaque-purified two or three times to ensure that one had a single virus. For the PCR DNA template preparation, $100 \mu \mathrm{L}$ of viral lysate was boiled in deionized sterile water for 5-10 min.

\subsection{Primer Selection}

DNA sequences 450 to $500 \mathrm{bp}$ upstream and 230 to $350 \mathrm{bp}$ downstream from the $k c v$ gene from 13 previously sequenced SAG chloroviruses [14] were used to identify conserved regions for designing degenerate primers. Conserved regions were identified inside the aligned sequences, and four forward primers and five reverse primers (Table 1) were made and tested using known SAG virus DNAs (ATCV-1, BRO604, Can0610, Canal1, GM0701, MN0810, MO0605, NEJV2, NTS1, OR0704, TN603, WI0606). The primers that identified all of the $k c v$ genes were forward primer Kcv8 Frw and reverse primer Kcv6 Rvs as well as forward primer Kcv9 Frw and reverse primer Kcv6 Rvs. As a result, the primer set Kcv9/Kcv6 was selected to amplify the $k c v$ genes.

Table 1. Primers tested to isolate the $k c v$ genes.

\begin{tabular}{llcccc}
\hline & Name & Sequence & Position & GC Content (\%) & $\mathbf{T}_{\mathbf{m}}\left({ }^{\circ} \mathbf{C}\right)$ \\
\hline \multirow{5}{*}{ Forward } & Kcv6 Frw & CTT TAG YYT TYY TCK GVC & -366 & 34 & 49 \\
Primers & Kcv7 Frw & CTT TAG YYT TYY TCK GVC G & -366 & 38 & 54 \\
& Kcv Frw & GAA GCA GGY ACC ACT TTA G & -379 & 47 & 53 \\
& Kcv Frw & GCA GGY ACC ACT TTA G & -376 & 50 & 47 \\
\hline \multirow{5}{*}{ Reverse } & Kcv6 Rvs & CRC RGM ATR TRT CAT TTG WCC C & +256 & 48 & 64 \\
Primers & Kcv7 Rvs & CTT ACR CRG MAT RTR TCA TTT G & +259 & 39 & 56 \\
& Kcv9 Rvs & CTT ACR CRG MAT RTR TC & +264 & 44 & 44 \\
& Kcv10 Rvs & CAT TTC TTA CRC RGM ATR TRT C & +262 & 39 & 45 \\
\hline
\end{tabular}

\subsection{Polymerase Chain Reactions (PCR)}

The conditions for the PCR reactions followed the recommendations of New England Biolab's (Beverly, MA, USA) Phusion High Fidelity DNA Polymerase kit. The $50 \mu \mathrm{L}$ PCR reactions contained $10 \mu \mathrm{L}$ of $5 \mathrm{x}$ Phusion High Fidelity buffer, 1 U Phusion DNA polymerase, and $1 \mu \mathrm{L}$ of DNA template. The final concentration of dNTPs in the PCR reaction was $0.2 \mathrm{mM}$ and the primer final concentration was $0.5 \mu \mathrm{M}$ forward primer and $0.5 \mu \mathrm{M}$ reverse primer. The reactions were run for $5 \mathrm{~min}$ at $94{ }^{\circ} \mathrm{C}$, followed by 35 cycles of $1 \mathrm{~min}$ at $94{ }^{\circ} \mathrm{C}, 30 \mathrm{~s}$ at $56{ }^{\circ} \mathrm{C}, 1 \mathrm{~min}$ at $72{ }^{\circ} \mathrm{C}$, and at the end the final extension $15 \mathrm{~min}$ at $72{ }^{\circ} \mathrm{C}$. Deionized water was used as a negative control.

The PCR products were gel-purified by mixing $20 \mu \mathrm{L}$ of an amplified sample with a small amount of Ficoll gel loading buffer and loaded on a 1\% agarose gel. The New England Biolabs log-2 ladder was used as a molecular weight marker. The gel was run at $5 \mathrm{~V} / \mathrm{cm}$ for $1 \mathrm{~h}$, then imaged by exposing the gel to ultraviolet light. Amplified $k c v$ genes were excised from the gel and the DNA extracted using the QIAquick Gel Extraction Kit following the manufacturer's instruction (QIAGEN Hilden, Germany). 
The purified DNAs were sequenced using Sanger sequencing by a commercial provider. The accession numbers for the $k c v$ sequences in GenBank are MT560092-MT560194.

\subsection{Phylogenetic Analysis}

The $k c v$ gene DNA sequences were translated into amino acid sequences, and all of the unique Kcv proteins were aligned. Geneious 11.0.5 software (Biomatters Ltd., Auckland, New Zealand, https: $/ / w w w . g e n e i o u s . c o m)$ was used for the DNA and protein sequence alignment (Geneious Alignment with the default settings) and the phylogenetic tree was constructed with PhyML (version 3.3.20180621), which is Maximum likelihood, using the default settings.

\subsection{Functional Reconstitution of kcv Genes in Planar Lipid Bilayers}

$\mathrm{K}^{+}$channel proteins were translated in vitro into nanodiscs (NDs) with the MembraneMax HN Protein Expression Kit (Invitrogen, Carlsbad, CA, USA) as described previously [15]. A His-tag attached to the scaffold protein of the NDs allowed purification of channel/ND-complexes via metal chelate affinity chromatography. To eliminate unspecific binders, the column was washed three times with $400 \mu \mathrm{L}$ of a $20 \mathrm{mM}$ imidazole solution. Finally, the His-tagged NDs were eluted in three fractions with $200 \mu \mathrm{L}$ of a $250 \mathrm{mM}$ imidazole solution. All centrifugation steps were performed at $700 \mathrm{~g}$ for 2 min.

Single-channel recordings were done with a vertical bilayer set up (IonoVation, Osnabrück, Germany) as described previously [16]. The experimental solution contained $100 \mathrm{mM}$ $\mathrm{KCl}$ and was buffered to $\mathrm{pH} 7.0$ with $10 \mathrm{mM} \mathrm{HEPES} / \mathrm{KOH}$. As a lipid, we used 1,2-diphythanoyl-sn-glycero-3-phosphocholine (DPhPC) (Avanti Polar Lipids, Alabaster, AL, USA) at a concentration of $15 \mathrm{mg} / \mathrm{mL}$ in n-pentane (MERCK KGaA, Darmstadt, Germany).

\section{Results}

\section{1. kcv Genes Selected for Analysis}

In total, $k c v$ genes from 83 SAG chloroviruses were obtained from the PCR experiments. In addition, the $k c v$ genes from the 13 SAG chloroviruses that had been sequenced previously [14] and $k c v$ genes from 7 recently sequenced SAG chloroviruses (not yet in the database) were included in the analysis. Thus, in total we compared $k c v$ genes from 103 SAG chloroviruses (Table A1). Overall, these viruses were from three continents and seven countries.

\subsection{Diversity of kcv Genes}

Alignment of the nucleotide sequences indicated that the $103 \mathrm{kcv}$ genes had substitutions in 125 of the 249 nucleotides ( 50\%) (assuming that all the proteins were 82 amino acids long (but see below)) producing 42 unique DNA sequences (Figure A1 (Appendix B)). Nine of the viruses with identical $k c v$ DNA sequences included a virus isolated in Germany in 2002 and viruses isolated in various parts of the United States of America 4 to 16 years later. Twenty of the virus isolates with identical $k c v$ DNA sequences were from ponds near Hohenheim, Germany that were collected on the same day in 2017. It is important to note that the type SAG virus, ATCV-1, was isolated from one of these ponds in 2002. The sequences of the newly isolated viruses differed from ATCV-1 virus by 18-21 amino acids. In these same recent German collections, there were viruses with three additional $k c v$ DNA sequences. The $k c v$ DNA sequence from 18 of the 103 viruses was only found one time.

\subsection{Diversity of the Kcv Proteins}

The 42 unique $k c v$ DNA sequences produced 26 unique proteins (Figure 2). Of the 26 unique protein sequences, 18 of them were 82 amino acids long, 4 were 84 amino acids, 2 were 85 amino acids, 1 was 87 amino acids, and 1 was 89 amino acids. The proteins with 84 and 85 amino acids had either 2 or 3 extra amino acids at their C-terminal ends. The 87 amino acid $\mathrm{Kcv}$ protein from a virus isolate 
from New York state had 5 amino acids added to the N-terminus of the protein. However, this protein has an internal Met that would create an 82 amino acid protein and so we suspect that this internal AUG is the actual translation start site. The 89 amino acid protein from a virus, GLND22, isolated in Greenland has 8 extra amino acids in the turret region between the outer transmembrane domain and the pore region. This turret region also has extra amino acids in the slightly larger Kcv proteins from the NC64A viruses (Figure 1) and the Pbi viruses [17].

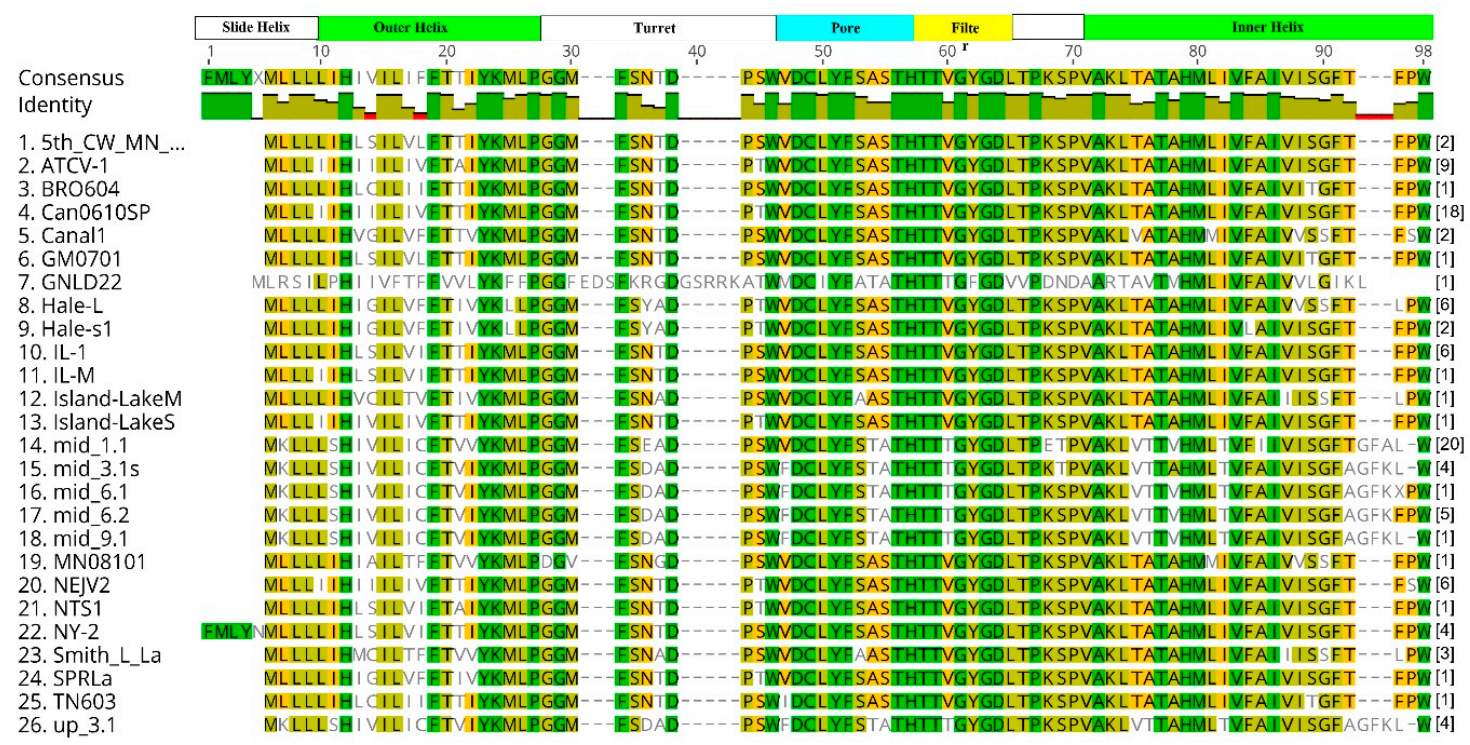

Figure 2. Amino acid alignment of SAG chlorovirus unique $\mathrm{Kcv}$ proteins using Geneious 11.0.5 software. The number in brackets at the end of each protein is the number of times that the protein had an identical sequence of the 103 viruses examined. Score matrix is Identity. Green color denotes identical amino acids. Other shades of amino acids indicate a level of conservation from olive color as being the most conserved to white color not conserved.

The $\mathrm{Kcv}_{\mathrm{Can} 0610 S P}$ only differed from $\mathrm{Kcv}_{\mathrm{ATCV}}-1$ by one amino acid. The Kcvs from viruses collected from Germany in 2017 differed from Kcv $\mathrm{ATCV}_{-1}$, which originally came from Germany, by 18 to 21 amino acids. The $\mathrm{Kcv}_{\mathrm{GNLD} 22}$ differed the most from $\mathrm{KCv}_{\mathrm{ATCV}}-1$ with 55 amino acid differences or $56 \%$ of the amino acids. The remaining virus Kcvs differed from Kcv $\mathrm{ATCV}_{-1}$ by 2 to 13 amino acids.

Alignment of the 26 unique proteins revealed that some areas of the Kcv protein were more conserved than others. The filter domain is typically the most highly conserved domain in $\mathrm{K}^{+}$channel proteins and 20 of the 26 proteins had a TTVGYGDL sequence. Five of the remaining six Kcvs had a TTTGYGDL sequence and the remaining $\mathrm{Kcv}, \mathrm{Kcv}_{\mathrm{GLND} 22}$ from Greenland, had a TTTGFGDV sequence (Figure 2). All the SAG chlorovirus Kcvs essentially lack an N-terminal slide helix domain (Figure 1).

A phylogenetic tree of the $26 \mathrm{Kcv}$ proteins resulted in 3 major clades (Figure 3). The largest clade had $19 \mathrm{Kcv}$ proteins from viruses primarily collected across the United States but also included viruses isolated in Canada, Guatemala, and Brazil, and one from Germany. Kcv proteins isolated from the recent German water samples formed a separate cluster with a distance of 0.4925 substitutions from the rest of the SAG Kcvs (Figure 3). The $\mathrm{Kcv}_{\text {GNDL22 }}$ from Greenland also formed a distinct clade with a distance of 1.518 substitutions from the German samples and a distance of 1.6675 substitutions from all the rest. Interestingly, $\mathrm{Kcv}_{\mathrm{GNDL} 22}$ had more similarity to a Kcv from a Pbi virus MT325 than it did to the other SAG viruses (Figure 3). Virus GNDL22 is also interesting because about $15 \%$ of its CDSs are more similar to the MT325 Pbi virus and the other $85 \%$ are most similar to SAG viruses. Thus, GNDL22 appears to be some type of hybrid virus. 


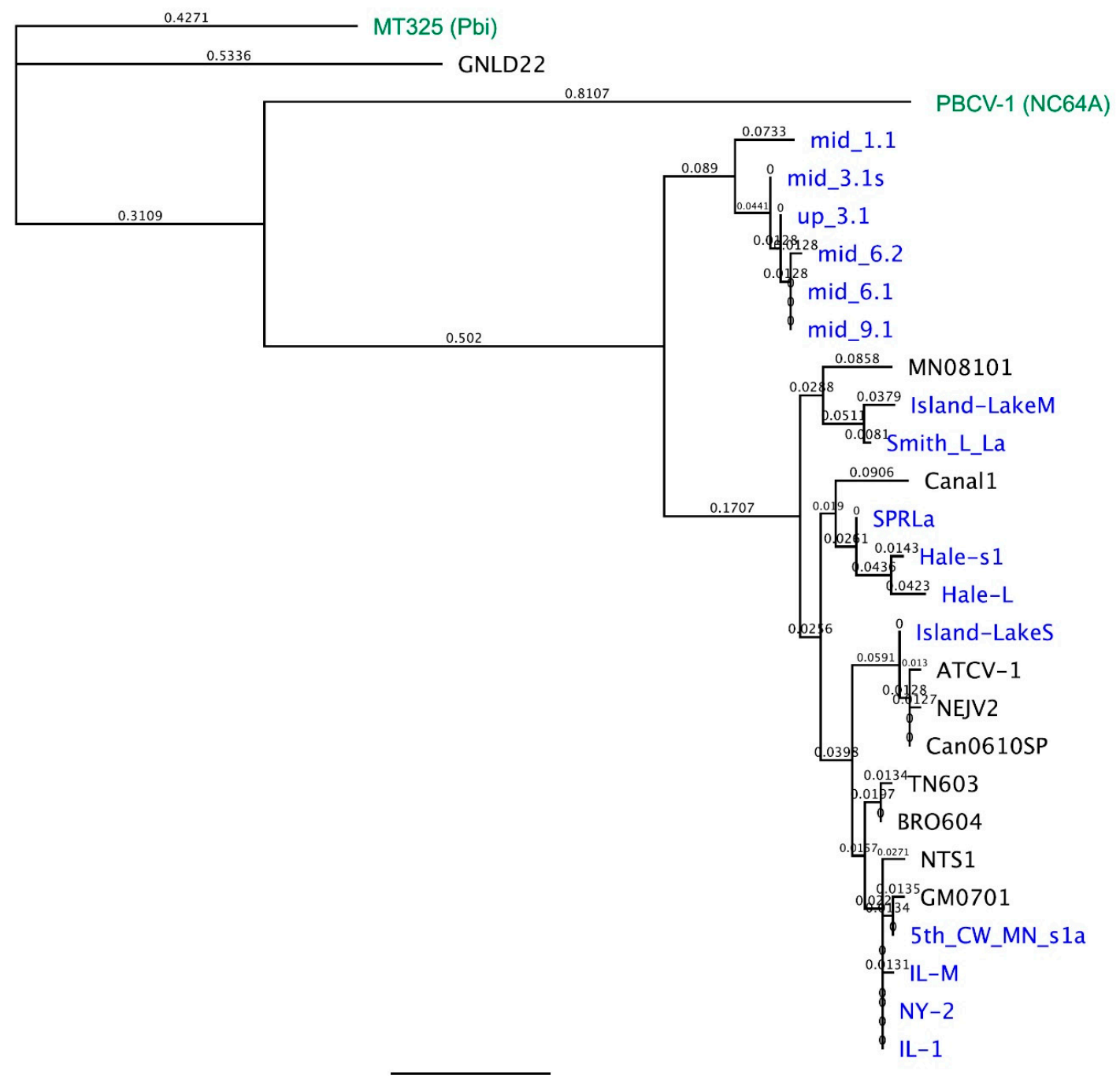

0.2

Figure 3. Phylogenetic tree of Kcv proteins from 26 SAG chloroviruses. PhyML, which is Maximum likelihood, with the default settings was used to construct the tree. The branch length shows dissimilarity between strains and the values on the branches are the number of changes. The viruses in blue represent the new Kcv proteins reported in this manuscript. Viruses MT325 representing a Pbi virus and PBCV-1 representing an NC64A virus are in green. Additional information on each of the Kcv proteins from the SAG viruses, including where they were isolated and the number of times that protein sequence appeared, is included in Table A1.

\subsection{Functional Reconstitution of Two New Kcv Channels in Planar Lipid Bilayers}

To test whether the newly discovered genes were coding for functional $\mathrm{K}^{+}$channels, we selected two representatives for functional testing. The putative channel proteins $\mathrm{Kcv}_{\mathrm{GNLD22}}$ and $\mathrm{Kcv}_{\mathrm{Can} 0610 \mathrm{SP}}$ were translated in vitro into nanodiscs and after purification reconstituted in planar lipid bilayers. The electrical recordings in Figure 4 show that the two proteins generated typical single-channel fluctuations at positive and negative voltages in the presence of $100 \mathrm{mM} \mathrm{KCl}$ on both sides of the membrane. These experiments established that the two genes code for functional ion channels. The overall properties of the two new channels were similar to those of $\mathrm{KCv}_{\mathrm{NTS}}$ (Figure 4), a well-studied representative of the $\mathrm{K}^{+}$channels from SAG viruses [18]; $\mathrm{Kcv}_{\mathrm{NTS}}$ differed from the reference channel $\mathrm{Kcv}_{\text {ATCV- } 1}$ by four amino acids. $\mathrm{Kcv}_{\mathrm{GNLD} 22}$ and $\mathrm{Kcv}_{\text {Can0610SP }}$ were selected because of their large (55 amino acids) and small ( 1 amino acid) deviation from the reference channel Kcv $\mathrm{ATCV}_{-1}$. All three channels exhibited a hallmark of the chlorovirus $\mathrm{K}^{+}$channels with well-resolved channel openings at 
positive voltages and flicker type gating at negative voltages. This flicker type gating resulted from very fast open/close transitions at negative voltages, which cannot be fully resolved by the recording equipment. As a consequence, the unitary channel conductance exhibited an apparent decrease at negative voltages $[19,20]$. However, it is interesting to note that this fast gating was already apparent in $\mathrm{Kcv}_{\mathrm{GNLD22}}$ at voltages negative of $0 \mathrm{mV}$ while the negative slope in the two other channels only occurred at voltages more negative than about $-100 \mathrm{mV}$. A closer scrutiny of the single-channel data also showed additional differences between the three channels. Comparison of the unitary channel conductance showed that this value in $\operatorname{Kcv}_{\text {GNLD22 }}(45 \pm 3 \mathrm{pS}, n=7)$ was only half as big as in $\mathrm{Kcv}_{\mathrm{NTS}}$ $(87 \pm 1.4 \mathrm{pS}, n=9)$ and $\mathrm{K}_{\mathrm{cv}}$ Can0610SP $(110 \pm 3, n=9)$. Another striking feature of $\mathrm{K}_{\mathrm{CV}} \mathrm{GNLD}_{22}$ was a strong voltage-dependent decrease in open probability at positive voltages, which was not seen in the other two. A peculiar feature of $\mathrm{Kcv}_{\text {Can0610SP }}$ was long-lived closed states at negative voltages, which explains a voltage-dependent decrease in open probability at negative voltages. These long closures were absent in the two other channels; in $\mathrm{Kcv}_{\mathrm{GNLD} 22}$ it was even difficult to observe any distinct closure at negative voltages.
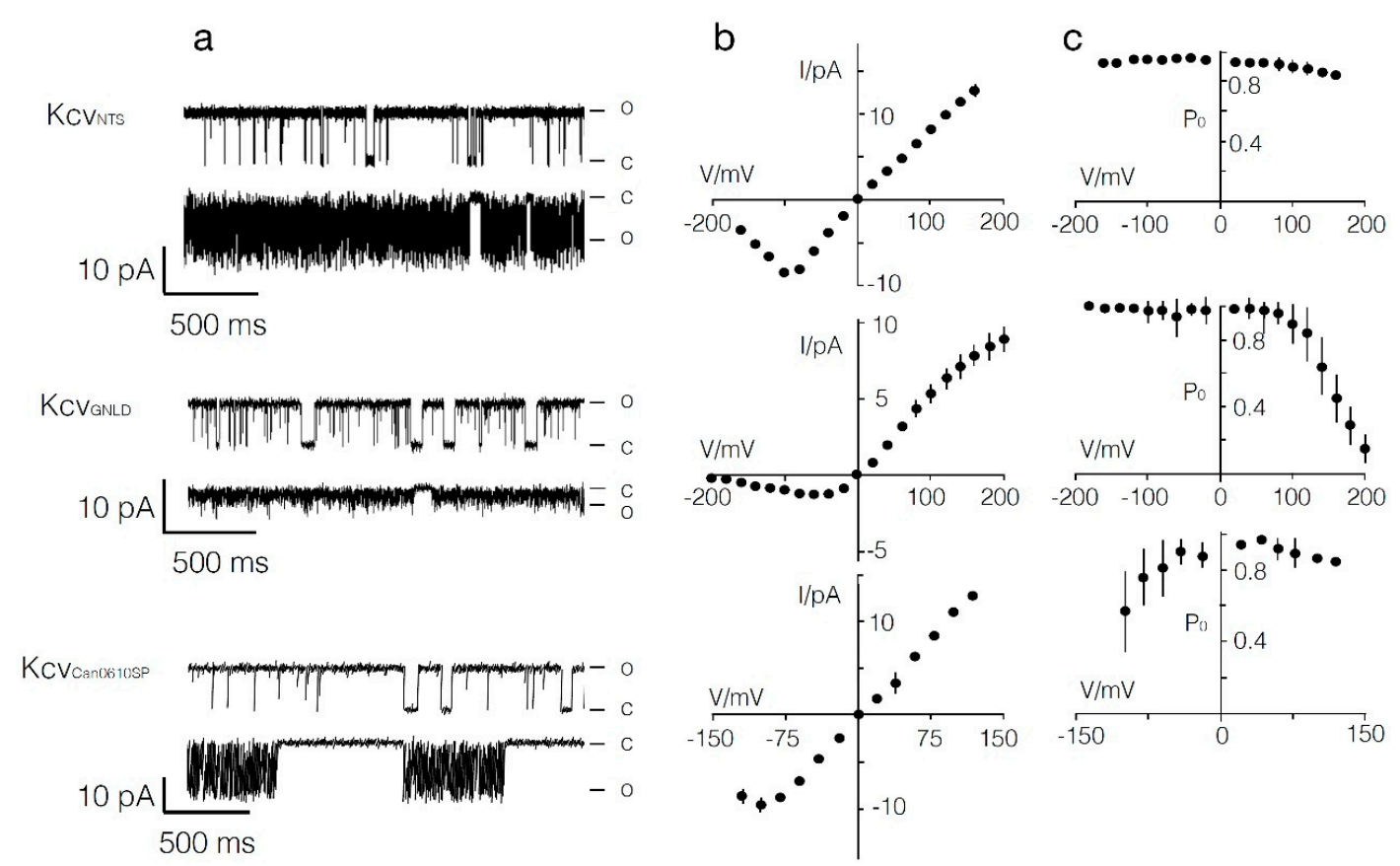

Figure 4. Two of the newly discovered $\mathrm{K}^{+}$channels are active. Characteristic single-channel fluctuations (a), mean single-channel I/V relations (b), and mean open probabilities (c) of $\mathrm{Kcv}_{\mathrm{NTS}}$ (top row), $\mathrm{Kcv}_{\mathrm{GNLD22}}$ (middle row), and $\mathrm{Kcv}_{\text {Can0610SP }}$ (bottom row) at $\pm 120 \mathrm{mV}$. The closed (c) and open (o) levels are indicated along the current traces. Data are means \pm s.d. from $\geq 3$ independent recordings of channels in the same row. Data were recorded in a DPhPC bilayer with symmetrical $100 \mathrm{mM} \mathrm{KCl}$, $10 \mathrm{mM}$ HEPES, pH 7 in cis and trans chamber.

\section{Discussion}

This manuscript demonstrated that the $k c v$ gene is ubiquitous among the 103 chloroviruses that infect $C$. heliozoae SAG 3.83, which resulted in 42 unique $k c v$ DNA sequences. The 42 unique $k c v$ DNA sequences produced 26 unique proteins or 26 new Kcv channels. Using the $\mathrm{Kcv}_{\mathrm{ATCV}-1}$ channel as representative of the SAG viruses, the Kcv ${ }_{\text {ATCV-1 }}$ differed from the $\mathrm{Kcv}_{\mathrm{GNLD} 22}$ channel from Greenland by 55 amino acids or $56 \%$ of their amino acids. Other channels differ from the Kcv ATCV- 1 channel by 1 to 21 amino acids.

Due to the role $\mathrm{K}^{+}$channels play in chlorovirus infection and reproduction by depolarizing the host cell membrane, it is not surprising that $k c v$ genes were found in all of the samples [19]. Therefore, we predict that all of the amino acid substitutions in the channels from the SAG viruses will produce 
functional channels; in fact, the two channels that were tested were functional (Figure 4). Furthermore, we predict that the SAG Kcv channels may have some different biophysical properties, especially the Kcv coded by the GNDL22 virus. This prediction is confirmed by scrutiny of the functional properties of the two new channels and a comparison with the well-studied SAG-type channel $\mathrm{Kcv}_{\mathrm{NTS}}$ [18]. The mutual comparisons identified differences in the unitary conductance and gating between the different channels. The apparent impact of a few amino acid exchanges between the proteins on functional properties is in good agreement with previous investigations in which we found that mutation of the common Gly at the end of the second transmembrane domain in $\mathrm{Kcv}_{\mathrm{NTS}}$ to Ser introduces one distinct gate with a long-lived close time [18]. Several of the new channels have the same critical Ser in the same position (90 in Figure 2). Thus, a functional analysis of these channels will be interesting.

The data are also in line with a previous study where the $k c v$ gene was sequenced from 41 NC64A viruses, including the prototype chlorovirus PBCV-1. Sixteen of the 94 amino acids in the NC64A KcV protein differed resulting in six new Kcv channels [20]. The six Kcv-like channels, which differed from $\mathrm{KCV}_{\mathrm{PBCV}-1}$ by 4 to 12 amino acids, produced $\mathrm{K}^{+}$selective currents in Xenopus laevis oocytes with altered biophysical properties, including current kinetics, voltage dependency, and inhibition by $\mathrm{Cs}^{+}[20,21]$. The amino acid changes together with the different properties observed in the six Kcv-like channels were used to guide site-directed mutations, either singularly or in combination, to identify key amino acids that confer specific properties to Kcv [20,21].

While we assume that the chloroviruses require $\mathrm{Kcv}$ activity to replicate, we do have to mention that out of the more than 150 chloroviruses that have been examined for a $k c v$ gene, two NC64A viruses either lack a $k c v$ gene or have a truncated form [1]. We would like to disrupt the $k c v$ gene in some of the chloroviruses to see what effect this has on virus replication, however, currently the technology to do this experiment is not available.

Compared with the larger KCV PBCV-1, the 82 amino acid $\mathrm{KCv}_{\mathrm{ATCV}-1}$ lacks a cytoplasmic $\mathrm{N}$-terminus, that is, the slide helix region and a number of charged amino acids in its turret domain (Figure 1) [12]. The only known $\mathrm{K}^{+}$channel proteins smaller than $\mathrm{Kcv}_{\text {ATCV- } 1}$ are two channels that are encoded by viruses that infect small marine algae in the Micromonas genus [22]. The channel $\mathrm{Kmbv}_{1}$ is 79 amino acids long and $\mathrm{Kmpv}_{12 \mathrm{~T}}$ is 78 amino acids long. Expression of $\mathrm{Kmbv}_{1}$ in HEK293 cells results in currents. However, expression of $\mathrm{Kmpv}_{12 \mathrm{~T}}$ in the same cells does not produce a current, but it does produce a current in a planar lipid bilayer [22]. All of these virus-encoded channels have a long evolutionary history and probably have a common evolutionary ancestor.

In summary, $k c v$ genes from 103 geographically distinct SAG viruses were sequenced to assess their genetic diversity. Of the $103 \mathrm{kcv}$ genes, there were 42 unique DNA sequences that translated into 26 new Kcv proteins, which we predict will have some different biophysical properties. The amino acid changes together with the expected different properties will be used to guide site-directed mutations to identify key amino acids that confer specific properties to Kcv.

Author Contributions: Conceptualization, J.L.V.E. and G.T.; methodology, C.R.M. and I.V.A.; software, C.R.M. and I.V.A.; validation, C.R.M., J.L.V.E., I.V.A., J.S.G., R.M.C., G.T., and B.H.; formal analysis, C.R.M. and G.T.; investigation, C.R.M., F.C.F., K.K., O.R., and B.H.; resources, C.R.M., I.V.A., F.C.F., J.S.G., R.M.C., and G.T.; data curation, C.R.M. and G.T.; writing-original draft preparation, C.R.M. and J.L.V.E.; writing-review and editing, C.R.M., J.L.V.E., I.V.A., R.C.C., F.C.F., J.S.G., G.T., B.H.; visualization, C.R.M., I.V.A., G.T.; supervision, J.L.V.E. and G.T.; project administration, J.L.V.E.; funding acquisition, J.L.V.E. and G.T. All authors have read and agreed to the published version of the manuscript.

Funding: This work was funded in part by the University of Nebraska-Lincoln's Undergraduate Creative Activities and Research Experience (UCARE) grant (C.R.M.), funding from the National Science Foundation under grant No. 1736030, (J.L.V.E.), and the European Research Council (ERC; 2015 Advanced Grant 495 (AdG) n. 695078 noMAGIC (G.T.).

Conflicts of Interest: The funders had no role in the design of the study; in the collection, analyses, or interpretation of data; in the writing of the manuscript, or in the decision to publish the results. 


\section{Appendix A}

Table A1. Source and sequence of SAG chlorovirus encoded potassium ion channel (Kcv) genes ${ }^{1}$.

\begin{tabular}{|c|c|c|c|c|c|}
\hline Sample ID & DNA Sequence & Location collected & Date collected & DNA Group & Amino Acid Group \\
\hline $\begin{array}{c}\text { 5th_CW_MN_s1a } \\
\text { (2) [2] }\end{array}$ & $\begin{array}{c}\text { ATGTTGCTGCTTCTCATACATCTCAGCATTTTGGT } \\
\text { ACTTTTCACTACCATATACAAGATGCTCCCCGGTGGC } \\
\text { ATGTTCTCGAACACGGATCCGTCCTGGGTCGATTGCC } \\
\text { TGTACTTTTCGGCATCAACGCACACCACCGTGGGGTA } \\
\text { CGGGGACCTCACGCCAAAATCACCCGTGGCAAAACT } \\
\text { CACGGCCACGGCACACATGCTGATCGTATTCGCGATC } \\
\text { GTCATTTCTGGCTTCACGTTCCCGTGGTAA }\end{array}$ & $\begin{array}{l}\text { 5th Crow Wing Lake; } \\
\text { Nevis, Minnesota }\end{array}$ & $5 / 4 / 2017$ & 5th_CW_MN_s1a & 5th_CW_MN_s1a \\
\hline 5th_CW_MN_L4 & $\begin{array}{c}\text { ATGTTGCTGCTTCTCATACATCTCAGCATTTTGGTA } \\
\text { CTTTTCACTACCATATACAAGATGCTCCCCGGTGGCAT } \\
\text { GTTCTCGAACACGGATCCGTCCTGGGTCGATTGCCTGT } \\
\text { ACTTTTCGGCATCAACGCACACCACCGTGGGGTACGGG } \\
\text { GACCTCACGCCAAAATCACCCGTGGCAAAACTCACGGC } \\
\text { CACGGCACACATGCTGATCGTATTCGCGATCGTCATTTCT } \\
\text { GGCTTCACGTTCCCGTGGTAA }\end{array}$ & $\begin{array}{l}\text { 5th Crow Wing Lake; } \\
\text { Nevis, Minnesota }\end{array}$ & $5 / 4 / 2017$ & 5th_CW_MN_s1a & 5th_CW_MN_s1a \\
\hline ATCV-1 (8) [9] & $\begin{array}{c}\text { ATGTTGCTGCTTATCATACATATCATCATTCTGATA } \\
\text { GTGTTCACTGCCATCTACAAGATGCTCCCCGGC } \\
\text { GGCATGTTCTCGAACACAGACCCTACTTGGGTT } \\
\text { GATTGCCTGTACTTTTCGGCATCGACGCACACC } \\
\text { ACCGTGGGGTACGGAGATCTCACGCCCAAATC } \\
\text { ACCCGTGGCAAAACTCACGGCAACGGCACAC } \\
\text { ATGTTGATCGTATTCGCGATCGTCATTTCTGGCT } \\
\text { TCACGTTTCCGTGGTAG }\end{array}$ & Germany & 2002 & ATCV-1 & ATCV-1 \\
\hline MO0605SPH & $\begin{array}{l}\text { ATGTTGCTGCTTATCATACATATCATCATTCTGATA } \\
\text { GTGTTCACTGCCATCTACAAGATGCTCCCCGGCG } \\
\text { GCATGTTCTCGAACACAGACCCTACTTGGGTTGA } \\
\text { TTGCCTGTACTTTTCGGCATCGACGCACACCACC } \\
\text { GTGGGGTACGGAGATCTCACGCCCAAATCACCCG } \\
\text { TGGCAAAACTCACGGCAACGGCACACATGTTGAT } \\
\text { CGTATTCGCGATCGTCATTTCTGGCTTCACGTTTCCGTGGTAG }\end{array}$ & Missouri & 2006 & ATCV-1 & ATCV-1 \\
\hline WI0606 & $\begin{array}{l}\text { ATGTTGCTGCTTATCATACATATCATCATTCTGATA } \\
\text { GTGTTCACTGCCATCTACAAGATGCTCCCCGGCG } \\
\text { GCATGTTCTCGAACACAGACCCTACTTGGGTTGA } \\
\text { TTGCCTGTACTTTTCGGCATCGACGCACACCACC } \\
\text { GTGGGGTACGGAGATCTCACGCCCAAATCACCCG } \\
\text { TGGCAAAACTCACGGCAACGGCACACATGTTGAT } \\
\text { CGTATTCGCGATCGTCATTTCTGGCTTCACGTTTCCGTGGTAG }\end{array}$ & Madison, Wisconsin & 2006 & ATCV-1 & ATCV-1 \\
\hline
\end{tabular}


Table A1. Cont.

\begin{tabular}{|c|c|c|c|c|c|}
\hline Sample ID & DNA Sequence & Location collected & Date collected & DNA Group & Amino Acid Group \\
\hline Drexel & $\begin{array}{l}\text { ATGTTGCTGCTTATCATACATATCATCATTCTG } \\
\text { ATAGTGTTCACTGCCATCTACAAGATGCTCCCCGGC } \\
\text { GGCATGTTCTCGAACACAGACCCTACTTGGGTTGAT } \\
\text { TGCCTGTACTTTTCGGCATCGACGCACACCACCGTG } \\
\text { GGGTACGGAGATCTCACGCCCAAATCACCCGTGGC } \\
\text { AAAACTCACGGCAACGGCACACATGTTGATCGTATT } \\
\text { CGCGATCGTCATTTCTGGCTTCACGTTTCCGTGGTAG }\end{array}$ & Drexel, Missouri & Jun-17 & ATCV-1 & ATCV-1 \\
\hline NPRLb & $\begin{array}{c}\text { ATGTTGCTGCTTATCATACATATCATCATTCTGATA } \\
\text { GTGTTCACTGCCATCTACAAGATGCTCCCCGGCGGC } \\
\text { ATGTTCTCGAACACAGACCCTACTTGGGTTGATTG } \\
\text { CCTGTACTTTTCGGCATCGACGCACACCACCGTG } \\
\text { GGGTACGGAGATCTCACGCCCAAATCACCCGTGG } \\
\text { CAAAACTCACGGCAACGGCACACATGTTGATCGT } \\
\text { ATTCGCGATCGTCATTTCTGGCTTCACGTTTCCGTGGTAG }\end{array}$ & $\begin{array}{c}\text { North Platte River; } \\
\text { Highway 27, Nebraska }\end{array}$ & $10 / 23 / 2017$ & ATCV-1 & ATCV-1 \\
\hline SPRma & $\begin{array}{c}\text { ATGTTGCTGCTTATCATACATATCATCATTCTGAT } \\
\text { AGTGTTCACTGCCATCTACAAGATGCTCCCCGG } \\
\text { CGGCATGTTCTCGAACACAGACCCTACTTGGGT } \\
\text { TGATTGCCTGTACTTTTCGGCATCGACGCACAC } \\
\text { CACCGTGGGGTACGGAGATCTCACGCCCAAAT } \\
\text { CACCCGTGGCAAAACTCACGGCAACGGCACA } \\
\text { CATGTTGATCGTATTCGCGATCGTCATTTCTGG } \\
\text { CTTCACGTTTCCGTGGTAG }\end{array}$ & $\begin{array}{l}\text { South Platte River; Big } \\
\text { Spring, Nebraska }\end{array}$ & $10 / 23 / 2017$ & ATCV-1 & ATCV-1 \\
\hline SPRsb & $\begin{array}{c}\text { ATGTTGCTGCTTATCATACATATCATCATTCTG } \\
\text { ATAGTGTTCACTGCCATCTACAAGATGCTCCCCGG } \\
\text { CGGCATGTTCTCGAACACAGACCCTACTTGGGTT } \\
\text { GATTGCCTGTACTTTTCGGCATCGACGCACACCA } \\
\text { CCGTGGGGTACGGAGATCTCACGCCCAAATCAC } \\
\text { CCGTGGCAAAACTCACGGCAACGGCACACATG } \\
\text { TTGATCGTATTCGCGATCGTCATTTCTGGCTTCA } \\
\text { CGTTTCCGTGGTAG }\end{array}$ & $\begin{array}{l}\text { South Platte River; Big } \\
\text { Spring, Nebraska }\end{array}$ & $10 / 23 / 2017$ & ATCV-1 & ATCV-1 \\
\hline
\end{tabular}


Table A1. Cont.

\begin{tabular}{|c|c|c|c|c|c|}
\hline Sample ID & DNA Sequence & Location collected & Date collected & DNA Group & Amino Acid Group \\
\hline $\begin{array}{l}\text { Smith-Lake_ } \\
\text { Large-Plaque }\end{array}$ & $\begin{array}{c}\text { ATGTTGCTGCTTATCATACATATCATCATTCTG } \\
\text { ATAGTGTTCACTGCCATCTACAAGATGCTCCCCGG } \\
\text { CGGCATGTTCTCGAACACAGACCCTACTTGGGTT } \\
\text { GATTGCCTGTACTTTTCGGCATCGACGCACACCA } \\
\text { CCGTGGGGTACGGAGATCTCACGCCCAAATCAC } \\
\text { CCGTGGCAAAACTCACGGCAACGGCACACATG } \\
\text { TTGATCGTATTCGCGATCGTCATTTCTGGCTTCA } \\
\text { CGTTTCCGTGGTAG }\end{array}$ & $\begin{array}{c}\text { Smith Lake, Western } \\
\text { Nebraska }\end{array}$ & 2018 & ATCV-1 & ATCV-1 \\
\hline OH-S (1) & $\begin{array}{c}\text { ATGTTGCTGCTTATCATACATATCATCATTCTGA } \\
\text { TAGTGTTCACTGCCATCTACAAGATGCTCCCCGGCG } \\
\text { GCATGTTCTCGAACACAGACCCTACTTGGGTTGATT } \\
\text { GCCTGTACTTTTCGGCATCGACGCACACCACCGT } \\
\text { GGGGTACGGAGATCTCACGCCCAAATCACCCGT } \\
\text { GGCAAAACTCACGGCAACGGCACACATGTTGAT } \\
\text { CGTATTCGCGATCGTCATTTCTGGCTTCACATTT } \\
\text { CCGTGGTAG }\end{array}$ & Ohio & Jul-17 & OH-S & ATCV-1 \\
\hline BRO604 (1) [1] & $\begin{array}{l}\text { ATGTTGCTGCTTCTCATACACCTCTGTATTCTGAT } \\
\text { AATTTTTACTACCATATACAAGATGTTGCCCGGAGG } \\
\text { CATGTTCTCTAACACGGACCCGTCGTGGGTCGATTG } \\
\text { CCTGTACTTCTCGGCATCAACGCACACCACCGTGGG } \\
\text { GTACGGGGATCTCACGCCCAAATCACCCGTGGCAA } \\
\text { AACTCACAGCAACGGCACACATGCTGATCGTATTCG } \\
\text { CGATCGTAATAACTGGCTTCACATTCCCGTGGTAA }\end{array}$ & Brazil & 2006 & BRO604 & BRO604 \\
\hline $\begin{array}{c}\text { Can0610SP (2) } \\
{[18]}\end{array}$ & $\begin{array}{c}\text { ATGTTGCTGCTTATCATACATATCATCATTCTGATA } \\
\text { GTGTTCACTACCATCTACAAGATGCTCCCCGGCGG } \\
\text { CATGTTCTCGAACACAGACCCTACTTGGGTTGATT } \\
\text { GCCTGTACTTTTCGGCATCGACGCACACCACTGT } \\
\text { GGGGTACGGAGATCTCACGCCCAAATCACCCGT } \\
\text { GGCAAAACTCACGGCAACGGCACACATGTTGA } \\
\text { TCGTATTCGCGATCGTCATTTCCGGCTTCACG } \\
\text { TTCCCGTGGTAG }\end{array}$ & British Columbia, Canada & 2006 & Can0610SP & Can0610SP \\
\hline
\end{tabular}


Table A1. Cont.

\begin{tabular}{|c|c|c|c|c|c|}
\hline Sample ID & DNA Sequence & Location collected & Date collected & DNA Group & Amino Acid Group \\
\hline Or0704 & $\begin{array}{c}\text { ATGTTGCTGCTTATCATACATATCATCATTCT } \\
\text { GATAGTGTTCACTACCATCTACAAGATGCTCCCCGGC } \\
\text { GGCATGTTCTCGAACACAGACCCTACTTGGGTTGAT } \\
\text { TGCCTGTACTTTTCGGCATCGACGCACACCACTGTG } \\
\text { GGGTACGGAGATCTCACGCCCAAATCACCCGTGGC } \\
\text { AAAACTCACGGCAACGGCACACATGTTGATCGTA } \\
\text { TTCGCGATCGTCATTTCCGGCTTCACGTTCCCGTGGTAG }\end{array}$ & $\begin{array}{l}\text { Willamette River; } \\
\text { Corvallis, Oregon }\end{array}$ & Jul-07 & Can0610SP & Can0610SP \\
\hline Chile_7s (1) & $\begin{array}{c}\text { ATGTTGCTGCTTATCATACATATCATCATTCTGATA } \\
\text { GTGTTCACTACCATATACAAGATGCTCCCCGGCGGCATG } \\
\text { TTCTCGAACACAGACCCTACTTGGGTTGATTGCCTGTACT } \\
\text { TTTCGGCATCGACGCACACCACTGTGGGGTACGGAGATCT } \\
\text { CACGCCCAAATCACCCGTGGCAAAACTCACGGCAACGGCA } \\
\text { CACATGTTGATCGTATTCGCGATCGTCATTTCCGGCTT } \\
\text { CACGTTCCCGTGGTAG }\end{array}$ & Chile & Jan-19 & Chile_7s & Can0610SP \\
\hline K2 (5) & $\begin{array}{l}\text { ATGTTGCTGCTTATCATACATATCATCATTCTGATAGTGTTC } \\
\text { ACTACCATCTACAAGATGCTCCCCGGTGGCATGTTCTCGAACA } \\
\text { CGGACCCGACTTGGGTTGATTGCCTGTACTTTTCGGCATCGA } \\
\text { CGCACACCACCGTGGGGTACGGAGATCTCACGCCCAAATC } \\
\text { ACCCGTGGCAAAACTCACGGCAACGGCACACATGTTGAT } \\
\text { CGTATTCGCGATCGTCATTTCCGGCTTCACGTTCCCGTGGTAG }\end{array}$ & $\begin{array}{c}\text { Missouri River; } \\
\text { Atchison, Kansas }\end{array}$ & $5 / 31 / 2017$ & $\mathrm{~K} 2$ & Can0610SP \\
\hline $\mathrm{K} 2 \mathrm{~s}$ & $\begin{array}{l}\text { ATGTTGCTGCTTATCATACATATCATCATTCTGATAGTGT } \\
\text { TCACTACCATCTACAAGATGCTCCCCGGTGGCATGTTCTCGA } \\
\text { ACACGGACCCGACTTGGGTTGATTGCCTGTACTTTTCGGCAT } \\
\text { CGACGCACACCACCGTGGGGTACGGAGATCTCACGCCCAA } \\
\text { ATCACCCGTGGCAAAACTCACGGCAACGGCACACATGTTG } \\
\text { ATCGTATTCGCGATCGTCATTTCCGGCTTCACGTTCCCGTGGTAG }\end{array}$ & $\begin{array}{l}\text { Missouri River; } \\
\text { Atchison, Kansas }\end{array}$ & $5 / 31 / 2017$ & $\mathrm{~K} 2$ & Can0610SP \\
\hline
\end{tabular}


Table A1. Cont.

\begin{tabular}{|c|c|c|c|c|c|}
\hline Sample ID & DNA Sequence & Location collected & Date collected & DNA Group & Amino Acid Group \\
\hline NPRma & $\begin{array}{c}\text { ATGTTGCTGCTTATCATACATATCATCATTCTGATAGTGTTC } \\
\text { ACTACCATCTACAAGATGCTCCCCGGTGGCATGTTCTCG } \\
\text { AACACGGACCCGACTTGGGTTGATTGCCTGTACTTTTC } \\
\text { GGCATCGACGCACACCACCGTGGGGTACGGAGATCTC } \\
\text { ACGCCCAAATCACCCGTGGCAAAACTCACGGCAACG } \\
\text { GCACACATGTTGATCGTATTCGCGATCGTCATTTCCG } \\
\text { GCTTCACGTTCCCGTGGTAG }\end{array}$ & $\begin{array}{c}\text { North Platte River; } \\
\text { Highway 27, Nebraska }\end{array}$ & $10 / 23 / 2017$ & K2 & Can0610SP \\
\hline NPRsb & $\begin{array}{c}\text { ATGTTGCTGCTTATCATACATATCATCATTCTGATAGTGTTC } \\
\text { ACTACCATCTACAAGATGCTCCCCGGTGGCATGTTCTCGA } \\
\text { ACACGGACCCGACTTGGGTTGATTGCCTGTACTTTTCGG } \\
\text { CATCGACGCACACCACCGTGGGGTACGGAGATCTCACG } \\
\text { CCCAAATCACCCGTGGCAAAACTCACGGCAACGGCAC } \\
\text { ACATGTTGATCGTATTCGCGATCGTCATTTCCGGCTTC } \\
\text { ACGTTCCCGTGGTAG }\end{array}$ & $\begin{array}{c}\text { North Platte River; } \\
\text { Highway 27, Nebraska }\end{array}$ & $10 / 23 / 2017$ & K2 & Can0610SP \\
\hline Pl_R_Lma & $\begin{array}{c}\text { ATGTTGCTGCTTATCATACATATCATCATTCTGATAGTGTTC } \\
\text { ACTACCATCTACAAGATGCTCCCCGGTGGCATGTTCTCGAA } \\
\text { CACGGACCCGACTTGGGTTGATTGCCTGTACTTTTCGGCAT } \\
\text { CGACGCACACCACCGTGGGGTACGGAGATCTCACGCCCA } \\
\text { AATCACCCGTGGCAAAACTCACGGCAACGGCACACATGT } \\
\text { TGATCGTATTCGCGATCGTCATTTCCGGCTTCA } \\
\text { CGTTCCCGTGGTAG }\end{array}$ & $\begin{array}{c}\text { Platte River; Louisville, } \\
\text { Nebraska }\end{array}$ & $10 / 26 / 2017$ & K2 & Can0610SP \\
\hline KS3-S (1) & $\begin{array}{c}\text { ATGTTGCTGCTTATCATACATATCATCATTCTGATAG } \\
\text { TGTTCACTACCATCTACAAGATGCTCCCCGGCGGCATG } \\
\text { TTCTCGAACACAGACCCTACTTGGGTCGATTGCCTGTA } \\
\text { CTTTTCGGCATCGACGCACACCACCGTGGGGTACGGAG } \\
\text { ATCTCACGCCCAAATCACCCGTGGCAAAACTCACGGCA } \\
\text { ACGGCACACATGTTGATCGTATTCGCGATCGTCATTT } \\
\text { CCGGCTTCACATTTCCGTGGTAA }\end{array}$ & $\begin{array}{c}\text { Delaware River; Valley } \\
\text { Fall, Kansas }\end{array}$ & Jun-17 & KS3-S & Can0610SP \\
\hline
\end{tabular}


Table A1. Cont.

\begin{tabular}{|c|c|c|c|c|c|}
\hline Sample ID & DNA Sequence & Location collected & Date collected & DNA Group & Amino Acid Group \\
\hline LP_CO_F1m (7) & $\begin{array}{c}\text { ATGTTGCTGCTTATCATACATATCATCATTCTGATAG } \\
\text { TGTTCACTACCATCTACAAGATGCTCCCCGGTGGCA } \\
\text { TGTTCTCGAACACGGACCCGACTTGGGTTGATTGCC } \\
\text { TGTACTTTTCGGCATCGACGCACACCACCGTGGGGT } \\
\text { ACGGAGATCTCACGCCCAAATCACCCGTGGCAAAA } \\
\text { CTCACGGCAACGGCACACATGTTGATCGTATTCGCGA } \\
\text { TCGTCATTTCCGGCTTCACGTTTCCGTGGTAA }\end{array}$ & $\begin{array}{c}\text { Cache la Poudre River, } \\
\text { Colorado }\end{array}$ & $5 / 29 / 2017$ & LP_CO_F1m & Can0610SP \\
\hline LP_CO_F2L & $\begin{array}{c}\text { ATGTTGCTGCTTATCATACATATCATCATTCTGATA } \\
\text { GTGTTCACTACCATCTACAAGATGCTCCCCGGTGGCAT } \\
\text { GTTCTCGAACACGGACCCGACTTGGGTTGATTGCCTGT } \\
\text { ACTTTTCGGCATCGACGCACACCACCGTGGGGTACGGA } \\
\text { GATCTCACGCCCAAATCACCCGTGGCAAAACTCACGGC } \\
\text { AACGGCACACATGTTGATCGTATTCGCGATCGTCATTTC } \\
\text { CGGCTTCACGTTTCCGTGGTAA }\end{array}$ & $\begin{array}{c}\text { Cache la Poudre River, } \\
\text { Colorado }\end{array}$ & $5 / 30 / 2017$ & LP_CO_F1m & Can0610SP \\
\hline LP_CO_F2m & $\begin{array}{l}\text { ATGTTGCTGCTTATCATACATATCATCATTCTGATAGTGTT } \\
\text { CACTACCATCTACAAGATGCTCCCCGGTGGCATGTTCTCGA } \\
\text { ACACGGACCCGACTTGGGTTGATTGCCTGTACTTTTCGGCA } \\
\text { TCGACGCACACCACCGTGGGGTACGGAGATCTCACGCCCA } \\
\text { AATCACCCGTGGCAAAACTCACGGCAACGGCACACATGTTG } \\
\text { ATCGTATTCGCGATCGTCATTTCCGGCTTCACGTTTCCGTGGTAA }\end{array}$ & $\begin{array}{l}\text { Cache la Poudre River, } \\
\text { Colorado } \\
\text { A }\end{array}$ & $5 / 31 / 2017$ & LP_CO_F1m & Can0610SP \\
\hline LP_CO_F2s & $\begin{array}{c}\text { ATGTTGCTGCTTATCATACATATCATCATTCTGATAGTGTT } \\
\text { CACTACCATCTACAAGATGCTCCCCGGTGGCATGTTCTC } \\
\text { GAACACGGACCCGACTTGGGTTGATTGCCTGTACTTTT } \\
\text { CGGCATCGACGCACACCACCGTGGGGTACGGAGATCT } \\
\text { CACGCCCAAATCACCCGTGGCAAAACTCACGGCAAC } \\
\text { GGCACACATGTTGATCGTATTCGCGATC } \\
\text { GTCATTTCCGGCTTCACGTTTCCGTGGTAA }\end{array}$ & $\begin{array}{c}\text { Cache la Poudre River, } \\
\text { Colorado }\end{array}$ & 6/1/2017 & LP_CO_F1m & Can0610SP \\
\hline LP_CO_F3L & $\begin{array}{c}\text { ATGTTGCTGCTTATCATACATATCATCATTCTGATAGTGTT } \\
\text { CACTACCATCTACAAGATGCTCCCCGGTGGCATGTTCTC } \\
\text { GAACACGGACCCGACTTGGGTTGATTGCCTGTACTTTT } \\
\text { CGGCATCGACGCACACCACCGTGGGGTACGGAGATCT } \\
\text { CACGCCCAAATCACCCGTGGCAAAACTCACGGCAAC } \\
\text { GGCACACATGTTGATCGTATTCGCGATCGTCATTTC } \\
\text { CGGCTTCACGTTTCCGTGGTAA }\end{array}$ & $\begin{array}{c}\text { Cache la Poudre River, } \\
\text { Colorado }\end{array}$ & 6/2/2017 & LP_CO_F1m & Can0610SP \\
\hline
\end{tabular}


Table A1. Cont.

\begin{tabular}{|c|c|c|c|c|c|}
\hline Sample ID & DNA Sequence & Location collected & Date collected & DNA Group & Amino Acid Group \\
\hline LP_CO_F3m & $\begin{array}{c}\text { ATGTTGCTGCTTATCATACATATCATCATTCTGATAGTGTTCA } \\
\text { CTACCATCTACAAGATGCTCCCCGGTGGCATGTTCTCGAAC } \\
\text { ACGGACCCGACTTGGGTTGATTGCCTGTACTTTTCGGCATC } \\
\text { GACGCACACCACCGTGGGGTACGGAGATCTCACGCCCAAA } \\
\text { TCACCCGTGGCAAAACTCACGGCAACGGCACACATGTTG } \\
\text { ATCGTATTCGCGATCGTCATTTCCGGCTTCACGTTTCC } \\
\text { GTGGTAA }\end{array}$ & $\begin{array}{l}\text { Cache la Poudre River, } \\
\text { Colorado }\end{array}$ & $6 / 3 / 2017$ & LP_CO_F1m & Can0610SP \\
\hline LP_CO_F4s & $\begin{array}{c}\text { ATGTTGCTGCTTATCATACATATCATCATTCTGATAGTGT } \\
\text { TCACTACCATCTACAAGATGCTCCCCGGTGGCATGTTCTC } \\
\text { GAACACGGACCCGACTTGGGTTGATTGCCTGTACTTTTCG } \\
\text { GCATCGACGCACACCACCGTGGGGTACGGAGATCTCACG } \\
\text { CCCAAATCACCCGTGGCAAAACTCACGGCAACGGCACA } \\
\text { CATGTTGATCGTATTCGCGATCGTCATTTC } \\
\text { CGGCTTCACGTTTCCGTGGTAA }\end{array}$ & $\begin{array}{l}\text { Cache la Poudre River, } \\
\text { Colorado }\end{array}$ & $6 / 4 / 2017$ & LP_CO_F1m & Can0610SP \\
\hline WR_DE (2) & $\begin{array}{c}\text { ATGTTGCTGCTTATCATACATATCATCATTCTGATA } \\
\text { GTGTTCACTACCATATACAAGATGCTCCCCGGCGGC } \\
\text { ATGTTCTCGAACACAGACCCTACTTGGGTCGATTGC } \\
\text { CTGTACTTTTCGGCATCGACGCACACCACCGTGGGG } \\
\text { TACGGAGATCTCACGCCCAAATCACCCGTGGCAAAA } \\
\text { CTCACGGCAACGGCGCACATGTTGATCGTATTCGCG } \\
\text { ATCGTCATTTCTGGATTCACGTTCCCGTGGTAG }\end{array}$ & $\begin{array}{l}\text { Wilson Run: Winterhur, } \\
\text { Delaware }\end{array}$ & Jul-17 & WR_DE & Can0610SP \\
\hline WR_DE_s2cr2 & $\begin{array}{l}\text { ATGTTGCTGCTTATCATACATATCATCATTCTGATAGT } \\
\text { GTTCACTACCATATACAAGATGCTCCCCGGCGGCAT } \\
\text { GTTCTCGAACACAGACCCTACTTGGGTCGATTGCC } \\
\text { TGTACTTTTCGGCATCGACGCACACCACCGTGGGG } \\
\text { TACGGAGATCTCACGCCCAAATCACCCGTGGCAAA } \\
\text { ACTCACGGCAACGGCGCACATGTTGATCGTATTCG } \\
\text { CGATCG TCATTTCTGGATTCACGTTCCCGTGGTAG }\end{array}$ & $\begin{array}{l}\text { Wilson Run, Winterthur; } \\
\text { Delaware }\end{array}$ & Jul-17 & WR_DE & Can0610SP \\
\hline Canal1 (2) [2] & $\begin{array}{c}\text { ATGTTGCTGCTCCTTATACACGTTGGTATTTTGGTATTTT } \\
\text { TCACCACCGTATACAAGATGCTCCCCGGTGGCATGTTC } \\
\text { TCGAATACGGACCCTAGCTGGGTAGATTGCTTATACTTC } \\
\text { TCAGCGTCAACTCACACCACCGTTGGGTACGGAGATC } \\
\text { TCACGCCCAATCACCCGTGGCGAAACTCGTGGCGAC } \\
\text { GGCGCATATGATGATCGTGTTCGCGATCGTTGTATCTAG } \\
\text { CTTCACGTTTTCGTGGTAG }\end{array}$ & $\begin{array}{c}\text { Canal exiting Smith Lake, } \\
\text { Nebraska }\end{array}$ & 2008 & Canal1 & Canal1 \\
\hline
\end{tabular}


Table A1. Cont.

\begin{tabular}{|c|c|c|c|c|c|}
\hline Sample ID & DNA Sequence & Location collected & Date collected & DNA Group & Amino Acid Group \\
\hline $\begin{array}{l}\text { Smith-Lake } \\
\text { Small-Plaque }\end{array}$ & $\begin{array}{c}\text { ATGTTGCTGCTCCTTATACACGTTGGTATTTTGGTATTTT } \\
\text { TCACCACCGTATACAAGATGCTCCCCGGTGGCATGTTCTCGA } \\
\text { TACGGACCCTAGCTGGGTAGATTGCTTATACTTCTCAGCGT } \\
\text { CAACTCACACCACCGTTGGGTACGGAGATCTCACGCCCAA } \\
\text { ATCACCCGTGGCGAAACTCGTGGCGACGGCGCATATGAT } \\
\text { GATCGTGTTCGCGATCGTTGTATCTAGCT } \\
\text { TCACGTTTTCGTGGTAG }\end{array}$ & $\begin{array}{l}\text { A } \\
\text { Smith Lake, Western } \\
\text { Nebraska }\end{array}$ & 2018 & Canal1 & Canal1 \\
\hline GM0701 (1) [1] & $\begin{array}{c}\text { ATGTTGCTGCTTCTCATACATCTCAGCATTTTGGTACTTTT } \\
\text { CACTACCATATACAAGATGCTCCCCGGCGCATGTTC } \\
\text { TCGAACACGGACCCATCCTGGGTCGATTGCCTGTAC } \\
\text { TTTTCAGCATCAACGCACACGACCGTGGGGTACGG } \\
\text { GGACCTCACGCCAAAATCGCCCGTGGCAAAACTC } \\
\text { ACAGCAACGGCACACATGCTGATCGTATTC } \\
\text { GCGATCGTAATAACTGGCTTCACATT CCCGTGGTAA }\end{array}$ & Guatemala & 2007 & GM0701 & GM0701 \\
\hline GNLD22 (1) [1] & $\begin{array}{c}\text { ATGCTGCGGTCAATATTGCCTCATATCATAGTGTTCAC } \\
\text { GTTTTTCGTTGTTCTTTACAAATTTTTCCCCGGGGG } \\
\text { TTTGAAGACTCATTCAAACGAGGAGACGGGTCCCGCA } \\
\text { GAAAGGCGACGTGGATGGACTGCATCTATTTCGCGACG } \\
\text { GCAACGCACACCACCACCGGGTTTGGTGATGTAGTCC } \\
\text { CCGACAACGACGCCGCAAGAACAGCTGTCACGATGC } \\
\text { ACATGCTCATAGTTTTCGCGATCGTAGTATT } \\
\text { GGGGATAAAACTCTAA }\end{array}$ & Lake Sisimiut, Greenland & 2012 & GNLD22 & GNLD22 \\
\hline Hale-L (2) [6] & $\begin{array}{c}\text { ATGTTGCTGCTCCTTATACACATCGGTATTTTGGTATTT } \\
\text { TTCACTATCGTGTACAAGCTGCTCCCTGGTGGCATGTT } \\
\text { CTCGTACGCAGACCCGACCTGGGTCGACTGCTTGTATT } \\
\text { TTTCGGCATCAACGCACACCACCGTGGGGTATGGGGAT } \\
\text { CTCACGCCCAAATCACCCGTGGCAAAACTCACGGCC } \\
\text { ACGGCACACATGCTGATTGTATTCGCGATCGTTGTCTC } \\
\text { TAGCTTTACGCTCCCCTGGTAA }\end{array}$ & $\begin{array}{l}\text { North branch of Elk } \\
\text { Creek; Hale, Wisconsin }\end{array}$ & $7 / 4 / 2017$ & Hale-L & Hale-L \\
\hline Hale_WI_m4 & $\begin{array}{c}\text { ATGTTGCTGCTCCTTATACACATCGGTATTTTGGTATTTTT } \\
\text { CACTATCGTGTACAAGCTGCTCCCTGGTGGCATGTTCTCGT } \\
\text { ACGCAGACCCGACCTGGGTCGACTGCTTGTATTTTTCGGCA } \\
\text { TCAACGCACACCACCGTGGGGTATGGGGATCTCACGCCCA } \\
\text { AATCACCCGTGGCAAAACTCACGGCCACGGCACACATGCT } \\
\text { GATTGTATTCGCGATCGTTGTCTCTAGCTTTAC } \\
\text { GCTCCCCTGGTAA }\end{array}$ & $\begin{array}{l}\text { North branch of Elk } \\
\text { Creek; Hale, Wisconsin }\end{array}$ & $7 / 4 / 2017$ & Hale-L & Hale-L \\
\hline
\end{tabular}


Table A1. Cont.

\begin{tabular}{|c|c|c|c|c|c|}
\hline Sample ID & DNA Sequence & Location collected & Date collected & DNA Group & Amino Acid Group \\
\hline TX3-L1 (4) & $\begin{array}{c}\text { ATGTTGCTGCTCCTTATACACATTGGTATTTTGGTATTTTTC } \\
\text { ACTATCGTGTACAAACTGCTCCCTGGTGGCATGTTCTCGT } \\
\text { ACGCAGATCCGACCTGGGTCGACTGCTTGTATTTTTCGGC } \\
\text { ATCAACGCACACCACCGTGGGGTATGGGGATCTCACGCC } \\
\text { CAAATCACCCGTGGCAAAACTCACGGCCACTGCACACA } \\
\text { TGCTGATTGTATTCGCGATCGTTGTCTCTAGCTTTAC } \\
\text { GCTCCCCTGGTAA }\end{array}$ & $\begin{array}{l}\text { Colorado River Pond; } \\
\text { Austin, Texas }\end{array}$ & Jul-17 & TX3-L1 & Hale-L \\
\hline TX3-L2 & $\begin{array}{c}\text { ATGTTGCTGCTCCTTATACACATTGGTATTTTGGTATTTTT } \\
\text { CACTATCGTGTACAAACTGCTCCCTGGTGGCATGTTCTC } \\
\text { GTACGCAGATCCGACCTGGGTCGACTGCTTGTATTTTTCG } \\
\text { GCATCAACGCACACCACCGTGGGGTATGGGGATCTCACG } \\
\text { CCCAAATCACCCGTGGCAAAACTCACGGCCACTGCACAC } \\
\text { ATGCTGATTGTATTCGCGATCGTTGTCTCTAGCTTTA } \\
\text { CGCTCCCCTGGTAA }\end{array}$ & $\begin{array}{l}\text { Colorado River Pond, } \\
\text { Austin, Texas }\end{array}$ & Jul-17 & TX3-L1 & Hale-L \\
\hline $\mathrm{TX} 3 \mathrm{~m}$ & 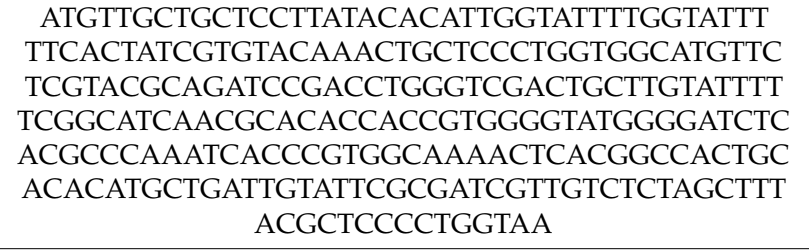 & $\begin{array}{l}\text { Colorado River Pond; } \\
\text { Austin, Texas }\end{array}$ & Jul-17 & TX3-L1 & Hale-L \\
\hline TX3s & $\begin{array}{l}\text { ATGTTGCTGCTCCTTATACACATTGGTATTTTGGTATTTTT } \\
\text { CACTATCGTGTACAAACTGCTCCCTGGTGGCATGTTCTC } \\
\text { GTACGCAGATCCGACCTGGGTCGACTGCTTGTATTTTTC } \\
\text { GGCATCAACGCACACCACCGTGGGGTATGGGGATCTCA } \\
\text { CGCCCAAATCACCCGTGGCAAAACTCACGGCCACTGC } \\
\text { ACACATGCTGATTGTATTCGCGATCGTTGTCTCTAGCTTT } \\
\text { ACGCTCCCCTGGTAA }\end{array}$ & $\begin{array}{l}\text { Colorado River Pond; } \\
\text { Austin, Texas }\end{array}$ & Jul-17 & TX3-L1 & Hale-L \\
\hline Hale-s1 (2) [2] & $\begin{array}{l}\text { ATGTTGCTGCTCCTTATACACATCGGTATTTTGGTATTTTT } \\
\text { CACTATCGTGTACAAGCTGCTCCCTGGTGGCATGTTCTCG } \\
\text { TACGCAGACCCGACCTGGGTCGACTGCTTGTATTTTTCGG } \\
\text { CATCAACGCACACCACCGTGGGGTATGGGGATCTCACGC } \\
\text { CCAAATCACCCGTGGCAAAACTCACGGCAACGGCACAC } \\
\text { ATGCTGATCGTACTCGCGATCGTCATTTCTGGCTTCACGT } \\
\text { TCCCGTGGTAG }\end{array}$ & $\begin{array}{l}\text { North branch of Elk } \\
\text { Creek; Hale, Wisconsin }\end{array}$ & $7 / 4 / 2017$ & Hale-s1 & Hale-s1 \\
\hline
\end{tabular}


Table A1. Cont.

\begin{tabular}{|c|c|c|c|c|c|}
\hline Sample ID & DNA Sequence & Location collected & Date collected & DNA Group & Amino Acid Group \\
\hline Hale-s2 & $\begin{array}{c}\text { ATGTTGCTGCTCCTTATACACATCGGTATTTTGGTATTTTTC } \\
\text { ACTATCGTGTACAAGCTGCTCCCTGGTGGCATGTTCTCGT } \\
\text { ACGCAGACCCGACCTGGGTCGACTGCTTGTATTTTTCGG } \\
\text { CATCAACGCACACCACCGTGGGGTATGGGGATCTCACGC } \\
\text { CCAAATCACCCGTGGCAAAACTCACGGCAACGGCACA } \\
\text { CATGCTGATCGTACTCGCGATCGTCATTTCTGGCTTCAC } \\
\text { GTTCCCGTGGTAG }\end{array}$ & $\begin{array}{l}\text { North branch of Elk } \\
\text { Creek; Hale, Wisconsin }\end{array}$ & $7 / 4 / 2017$ & Hale-s1 & Hale-s1 \\
\hline IL-1 (1) [6] & $\begin{array}{c}\text { ATGTTGCTGCTTCTCATACATCTCAGCATTTTGGTAATT } \\
\text { TTCACTACCATATACAAGATGCTCCCCGGCGGCATGT } \\
\text { TCTCGAACACGGATCCGTCCTGGGTCGATTGCCTGTA } \\
\text { CTTTTCGGCATCAACGCACACCACCGTGGGGTACGG } \\
\text { GGACCTCACGCCAAAATCACCCGTGGCAAAACTCA } \\
\text { CGGCAACGGCACACATGCTGATCGTATTTGCGATCGT } \\
\text { CATTTCTGGCTTCACGTTCCCGTGGTAA }\end{array}$ & Lake Zurich, Illinois & $6 / 27 / 2017$ & IL-1 & IL-1 \\
\hline Dismal_NE (1) & $\begin{array}{l}\text { ATGTTGCTGCTTCTCATACATCTCAGCATTTTGGTA } \\
\text { ATTTTCACTACCATCTACAAGATGCTCCCCGGCGGC } \\
\text { ATGTTCTCGAACACGGACCCATCCTGGGTCGATTGC } \\
\text { CTGTACTTTTCGGCATCAACGCACACCACCGTGGG } \\
\text { GTACGGGGACCTCACGCCAAAATCACCCGTGGA } \\
\text { AAACTCACGGCAACGGCACACATGCTGATCGTATT } \\
\text { CGCGATCGTCATTTCTGGCTTCACGTTCCCGTGGTAG }\end{array}$ & Dismal River, Nebraska & $5 / 26 / 2017$ & Dismal_NE & IL-1 \\
\hline NY2s1 (2) & $\begin{array}{c}\text { ATGTTGCTGCTTCTCATACATCTCAGCATTTTGGTAATTTTCA } \\
\text { CTACCATCTACAAGATGCTCCCCGGCGGCATGTTCTCGAAC } \\
\text { ACGGACCCATCCTGGGTCGATTGCCTGTACTTTTCGGCAT } \\
\text { CAACGCACACCACCGTGGGGTACGGGGACCTCACGCCCA } \\
\text { AATCACCCGTTGCAAAACTCACGGCAACGGCACACATGC } \\
\text { TGATCGTATTCGCGATCGTCATTTCTGGCTTCACGTT } \\
\text { CCCGTGGTAA }\end{array}$ & $\begin{array}{c}\text { Moodna Creek; } \\
\text { Washingtonville, New } \\
\text { York }\end{array}$ & $6 / 21 / 2017$ & NY-2s1 & IL-1 \\
\hline$N Y-2 s 2$ & $\begin{array}{l}\text { ATGTTGCTGCTTCTCATACATCTCAGCATTTTGGTAAT } \\
\text { TTTCACTACCATCTACAAGATGCTCCCCGGCGGCAT } \\
\text { GTTCTCGAACACGGACCCATCCTGGGTCGATTGCCT } \\
\text { GTACTTTTCGGCATCAACGCACACCACCGTGGGGT } \\
\text { ACGGGGACCTCACGCCCAAATCACCCGTTGCAAAA } \\
\text { CTCACGGCAACGGCACACATGCTGATCGTATTCGC } \\
\text { GATCGTCATTTCTGGCTTCACGTTCCCGTGGTAA }\end{array}$ & $\begin{array}{c}\text { Moodna Creek; } \\
\text { Washingtonville, New } \\
\text { York }\end{array}$ & $6 / 21 / 2017$ & NY-2s1 & IL-1 \\
\hline
\end{tabular}


Table A1. Cont.

\begin{tabular}{|c|c|c|c|c|c|}
\hline Sample ID & DNA Sequence & Location collected & Date collected & DNA Group & Amino Acid Group \\
\hline Pl_R_OLa (1) & $\begin{array}{l}\text { ATGTTGCTGCTTCTCATACATCTCAGCATTTTGGTA } \\
\text { ATTTTCACTACCATCTACAAGATGCTCCCCGGGG } \\
\text { CATGTTCTCGAACACGGACCCATCCTGGGTCGAT } \\
\text { TGCCTGTACTTTTCGGCATCAACGCACACCACCGT } \\
\text { GGGGTACGGGGACCTCACGCCAAAATCACCCGTG } \\
\text { GCAAAACTCACGGCAACGGCACACATGCTGATCG } \\
\text { TATTCGCGATCGTCATTTCTGGCTTCACGTTCCCATGGTAG }\end{array}$ & $\begin{array}{c}\text { Platte River; Odessa, } \\
\text { Nebraska }\end{array}$ & $10 / 23 / 2017$ & Pl_R_Ola & IL-1 \\
\hline $\begin{array}{c}\text { Somers_MT_m1 } \\
(1)\end{array}$ & $\begin{array}{l}\text { ATGTTGCTGCTTCTCATACATCTCAGCATTTTGGTA } \\
\text { ATTTTCACTACCATATACAAGATGCTCCCCGGCGGC } \\
\text { ATGTTCTCAAACACGGATCCGTCCTGGGTCGATTGC } \\
\text { CTGTACTTTTCGGCATCAACGCACACCACCGTGGG } \\
\text { GTACGGGGACCTCACGCCAAAATCACCCGTGGCAA } \\
\text { AACTCACGGCAACGGCACACATGCTGATCGTATTC } \\
\text { GCGATCGTCATTTCTGGCTTCACGTTCCCGTGGTAA } \\
\end{array}$ & $\begin{array}{c}\text { Flathead Lake; Somers, } \\
\text { Montana }\end{array}$ & 6/27/2017 & Somers_MT_m1 & IL-1 \\
\hline IL-M (1) [1] & $\begin{array}{c}\text { ATGTTGCTGCTTATCATACATCTCAGCATTTTGGTAA } \\
\text { TTTTCACTACCATATACAAGATGCTCCCCGGCGGCA } \\
\text { TGTTCTCGAACACGGATCCGTCCTGGGTCGATTGCC } \\
\text { TGTACTTTTCGGCATCAACGCACACCACCGTGGGGT } \\
\text { ACGGGGACCTCACGCCAAAATCACCCGTGGCAAAA } \\
\text { CTCACGGCAACGGCACACATGCTGATCGTATTTGCG } \\
\text { ATCGTCATTTCTGGCTTCACATTTCCGTGGTAG }\end{array}$ & Lake Zurich, Illinois & $6 / 27 / 2017$ & IL-M & IL-M \\
\hline $\begin{array}{l}\text { Island-Lake__ } \\
\text { Medium (1) [1] }\end{array}$ & $\begin{array}{c}\text { ATGTTGCTGCTCCTTATCCACGTGTGTATTTTGACAGTC } \\
\text { TTCACGATTGTTACAAGATGCTCCCTGGCGGCATGTTC } \\
\text { TCTAACGCGGACCCGTCGTGGGTAGACTGCTTATACTTT } \\
\text { GCCGCGTCGACTCACACCACAGTGGGGTACGGGGACC } \\
\text { TCACCCCCAAATCGCCAGTGGCAAAGCTCACGGCGAC } \\
\text { GGCCCACATGTTGATCGTGTTCGCGATCATTATATC } \\
\text { TAGCTTCACACTGCCATGGTAG } \\
\text {. }\end{array}$ & $\begin{array}{c}\text { Island Lake, Western } \\
\text { Nebraska }\end{array}$ & 2018 & Island-Lake_Medium & Island-Lake_Medium \\
\hline $\begin{array}{l}\text { Island-Lake_Small } \\
\text { (1) [1] }\end{array}$ & $\begin{array}{c}\text { ATGTTGCTGCTTATCATACATATCGTCATTCTTATAGTG } \\
\text { TTCACTACCATCTACAAGATGCTCCCCGGCGGCATGTT } \\
\text { CTCGAACACGGACCCGACTTGGGTTGATTGCCTGTACT } \\
\text { TTTCGGCATCGACGCACACCACCGTGGGGTACGGAGA } \\
\text { TCTCACGCCCAAATCACCCGTGGCAAAGCTCACGGCA } \\
\text { ACGGCACACATGCTGATCGTATTCGCGATCGTCATTTCT } \\
\text { GGCTTCACGTTTCCGTGGTAG }\end{array}$ & $\begin{array}{c}\text { Island Lake, Western } \\
\text { Nebraska }\end{array}$ & 2018 & Island-Lake_Small & Island-Lake_Small \\
\hline
\end{tabular}


Table A1. Cont.

\begin{tabular}{|c|c|c|c|c|c|}
\hline Sample ID & DNA Sequence & Location collected & Date collected & DNA Group & Amino Acid Group \\
\hline mid_1.1 (1) [20] & $\begin{array}{c}\text { ATGAAGCTGCTACTTTCACATATTGTTATTCTAATATGTT } \\
\text { TCACCGTCGTGTACAAGATGCTCCCCGGTGGCATGTTC } \\
\text { TCGGAAGCAGACCCGTCGTGGGTTGACTGTCTGTATTT } \\
\text { CTCGACGGCAACACACACGACAACGGGCTACGGCGAT } \\
\text { CTAACGCCAGAAACCCCGGTGGCAAAACTCGTGACAA } \\
\text { CGGTGCACATGTTAACCGTGTTCATCATCGTTATTTCCG } \\
\text { GCTTCACTGGCTTCGCATTATGGTAG }\end{array}$ & Germany & Oct-17 & mid_1.1 & mid_1.1 \\
\hline up_1.2m1 (19) & $\begin{array}{c}\text { ATGAAGCTGCTACTTTCACATATCGTTATTCTAATATGTTT } \\
\text { CACCGTCGTGTACAAGATGCTCCCCGGTGGCATGTTCT } \\
\text { CGGAAGCAGACCCGTCGTGGGTTGACTGTCTGTATTTC } \\
\text { TCGACGGCAACACACACGACAACGGGCTACGGCGATC } \\
\text { TAACGCCAGAAACCCCGGTGGCAAAACTCGTGACAAC } \\
\text { GGTGCACATGTTAACCGTGTTCATCATCGTTATTTCCGG } \\
\text { CTTCACTGGCTTCGCATTATGGTAG } \\
\end{array}$ & Germany & Oct-17 & up_1.2m1 & mid_1.1 \\
\hline mid_1.2m & $\begin{array}{c}\text { ATGAAGCTGCTACTTTCACATATCGTTATTCTAATATGTT } \\
\text { TCACCGTCGTGTACAAGATGCTCCCCGGTGGCATGTTCT } \\
\text { CGGAAGCAGACCCGTCGTGGGTTGACTGTCTGTATTTCT } \\
\text { CGACGGCAACACACACGACAACGGGCTACGGCGATCTA } \\
\text { ACGCCAGAAACCCCGGTGGCAAAACTCGTGACAACGG } \\
\text { TGCACATGTTAACCGTGTTCATCATCGTTATTTCCGGCTTC } \\
\text { ACTGGCTTCGCATTATGGTAG }\end{array}$ & Germany & Oct-17 & up_1.2m1 & mid_1.1 \\
\hline mid_1.2s2 & $\begin{array}{l}\text { ATGAAGCTGCTACTTTCACATATCGTTATTCTAATATGTTTC } \\
\text { ACCGTCGTGTACAAGATGCTCCCCGGTGGCATGTTCTCGGA } \\
\text { AGCAGACCCGTCGTGGGTTGACTGTCTGTATTTCTCGACG } \\
\text { GCAACACACACGACAACGGGCTACGGCGATCTAACGCCA } \\
\text { GAAACCCCGGTGGCAAAACTCGTGACAACGGTGCACATG } \\
\text { TTAACCGTGTTCATCATCGTTATTTCCGGCTTCACTGGCTT } \\
\text { CGCATTATGGTAG }\end{array}$ & Germany & Oct-17 & up_1.2m1 & mid_1.1 \\
\hline mid_10.1L & $\begin{array}{l}\text { ATGAAGCTGCTACTTTCACATATCGTTATTCTAATATGTTTC } \\
\text { ACCGTCGTGTACAAGATGCTCCCCGGTGGCATGTTCTCGG } \\
\text { AAGCAGACCCGTCGTGGGTTGACTGTCTGTATTTCTCGAC } \\
\text { GGCAACACACACGACAACGGGCTACGGCGATCTAACGCC } \\
\text { AGAAACCCCGGTGGCAAAACTCGTGACAACGGTGCACAT } \\
\text { GTTAACCGTGTTCATCATCGTTATT } \\
\text { TCCGGCTTCACTGGCTTCGCATTATGGTAG }\end{array}$ & Germany & Oct-17 & up_1.2m1 & mid_1.1 \\
\hline
\end{tabular}


Table A1. Cont.

\begin{tabular}{|c|c|c|c|c|c|}
\hline Sample ID & DNA Sequence & Location collected & Date collected & DNA Group & Amino Acid Group \\
\hline mid_10.1s1 & $\begin{array}{l}\text { ATGAAGCTGCTACTTTCACATATCGTTATTCTAATATGTTTC } \\
\text { ACCGTCGTGTACAAGATGCTCCCCGGTGCATGTTCTCGG } \\
\text { AAGCAGACCCGTCGTGGGTTGACTGTCTGTATTTCTCGAC } \\
\text { GGCAACACACACGACAACGGGCTACGGCGATCTAACGCC } \\
\text { AGAAACCCCGGTGGCAAAACTCGTGACAACGGTGCACA } \\
\text { TGTTAACCGTGTTCATCATCGTTATTTCCGGCTTCACTGGC } \\
\text { TTCGCATTATGGTAG }\end{array}$ & Germany & Oct-17 & up_1.2m1 & mid_1.1 \\
\hline mid_10.1s2 & $\begin{array}{c}\text { ATGAAGCTGCTACTTTCACATATCGTTATTCTAATATGTTTC } \\
\text { ACCGTCGTGTACAAGATGCTCCCCGGTGGCATGTTCTCG } \\
\text { GAAGCAGACCCGTCGTGGGTTGACTGTCTGTATTTCTCG } \\
\text { ACGGCAACACACACGACAACGGGCTACGGCGATCTAAC } \\
\text { GCCAGAAACCCCGGTGGCAAAACTCGTGACAACGGTG } \\
\text { CACATGTTAACCGTGTTCATCATCGTTATTTCCGGCTTCA } \\
\text { CTGGCTTCGCATTATGGTAG }\end{array}$ & Germany & Oct-17 & up_1.2m1 & mid_1.1 \\
\hline mid_11.1m & $\begin{array}{l}\text { ATGAAGCTGCTACTTTCACATATCGTTATTCTAATATGTTT } \\
\text { CACCGTCGTGTACAAGATGCTCCCCGGTGGCATGTTCTCGGA } \\
\text { AGCAGACCCGTCGTGGGTTGACTGTCTGTATTTCTCGACGG } \\
\text { CAACACACACGACAACGGGCTACGGCGATCTAACGCCAG } \\
\text { AAACCCCGGTGGCAAAACTCGTGACAACGGTGCACATGT } \\
\text { TAACCGTGTTCATCATCGTTATTTCCGGCTTCACTGGCTTCG } \\
\text { CATTATGGTAG }\end{array}$ & Germany & Oct-17 & up_1.2m1 & mid_1.1 \\
\hline mid_13.1L1 & $\begin{array}{l}\text { ATGAAGCTGCTACTTTCACATATCGTTATTCTAATATGTTTC } \\
\text { ACCGTCGTGTACAAGATGCTCCCCGGTGGCATGTTCTCGG } \\
\text { AAGCAGACCCGTCGTGGGTTGACTGTCTGTATTTCTCGAC } \\
\text { GGCAACACACACGACAACGGGCTACGGCGATCTAACGCC } \\
\text { AGAAACCCCGGTGGCAAAACTCGTGACAACGGTGCACA } \\
\text { TGTTAACCGTGTTCATCATCGTTATTCCGGCTTCACTGGC } \\
\text { TTCGCATTATGGTAG }\end{array}$ & Germany & Oct-17 & up_1.2m1 & mid_1.1 \\
\hline mid_13.1L2 & $\begin{array}{c}\text { ATGAAGCTGCTACTTTCACATATCGTTATTCTAATATGTTT } \\
\text { CACCGTCGTGTACAAGATGCTCCCCGGTGGCATGTTCTC } \\
\text { GGAAGCAGACCCGTCGTGGGTTGACTGTCTGTATTTCTC } \\
\text { GACGGCAACACACACGACAACGGGCTACGGCGATCTAA } \\
\text { CGCCAGAAACCCCGTGGCAAAACTCGTGACAACGGT } \\
\text { GCACATGTTAACCGTGTTCATCATCGTTATTTCCGGCTTC } \\
\text { ACTGGCTTCGCATTATGGTAG }\end{array}$ & Germany & Oct-17 & up_1.2m1 & mid_1.1 \\
\hline
\end{tabular}


Table A1. Cont.

\begin{tabular}{|c|c|c|c|c|c|}
\hline Sample ID & DNA Sequence & Location collected & Date collected & DNA Group & Amino Acid Group \\
\hline mid_13.1s1 & $\begin{array}{c}\text { ATGAAGCTGCTACTTTCACATATCGTTATTCTAATATGTTT } \\
\text { CACCGTCGTGTACAAGATGCTCCCCGGTGGATGTTCTC } \\
\text { GGAAGCAGACCCGTCGTGGGTTGACTGTCTGTATTTCTC } \\
\text { GACGGCAACACACACGACAACGGGCTACGGCGATCTAA } \\
\text { CGCCAGAAACCCGGTGGCAAAACTCGTGACAACGGTG } \\
\text { CACATGTTAACCGTGTTCATCATCGTTATTTCCGGCTTCAC } \\
\text { TGGCTTCGCATTATGGTAG }\end{array}$ & Germany & Oct-17 & up_1.2m1 & mid_1.1 \\
\hline mid_5.1L1 & $\begin{array}{c}\text { ATGAAGCTGCTACTTTCACATATCGTTATTCTAATATGTTTC } \\
\text { ACCGTCGTGTACAAGATGCTCCCCGGTGGCATGTTCTCG } \\
\text { GAAGCAGACCCGTCGTGGGTTGACTGTCTGTATTTCTCG } \\
\text { ACGGCAACACACACGACAACGGGCTACGGCGATCTAA } \\
\text { CGCCAGAAACCCCGTGGCAAAACTCGTGACAACGGT } \\
\text { GCACATGTTAACCGTGTTCATCATCGTTATTTCCGGCTTC } \\
\text { ACTGGCTTCGCATTATGGTAG }\end{array}$ & Germany & Oct-17 & up_1.2m1 & mid_1.1 \\
\hline mid_5.1L2 & $\begin{array}{c}\text { ATGAAGCTGCTACTTTCACATATCGTTATTCTAATATGTT } \\
\text { TCACCGTCGTGTACAAGATGCTCCCCGGTGGCATGTTCT } \\
\text { CGGAAGCAGACCCGTCGTGGGTTGACTGTCTGTATTTCT } \\
\text { CGACGGCAACACACACGACAACGGGCTACGGCGATCTA } \\
\text { ACGCCAGAAACCCCGGTGGCAAAACTCGTGACAACGG } \\
\text { TGCACATGTTAACCGTGTTCATCATCGTTATTTCCGGCTT } \\
\text { CACTGGCTTCGCATTATGGTAG }\end{array}$ & Germany & Oct-17 & up_1.2m1 & mid_1.1 \\
\hline mid_5.1s1 & $\begin{array}{l}\text { ATGAAGCTGCTACTTTCACATATCGTTATTCTAATATGTTTC } \\
\text { ACCGTCGTGTACAAGATGCTCCCCGGTGGCATGTTCTCGG } \\
\text { AAGCAGACCCGTCGTGGGTTGACTGTCTGTATTTCTCGAC } \\
\text { GGCAACACACACGACAACGGGCTACGGCGATCTAACGCC } \\
\text { AGAAACCCCGTGGCAAAACTCGTGACAACGGTGCACAT } \\
\text { GTTAACCGTGTTCATCATCGTTATTTCCGGCTTCACTGGCT } \\
\text { TCGCATTATGGTAG }\end{array}$ & Germany & Oct-17 & up_1.2m1 & mid_1.1 \\
\hline mid_7.2 & $\begin{array}{l}\text { ATGAAGCTGCTACTTTCACATATCGTTATTCTAATATGTTTCACCG } \\
\text { TCGTGTACAAGATGCTCCCCGGTGGCATGTTCTCGGAAGCAGAC } \\
\text { CCGTCGTGGGTTGACTGTCTGTATTTCTCGACGGCAACACACAC } \\
\text { GACAACGGGCTACGGCGATCTAACGCCAGAAACCCCGGTGGC } \\
\text { AAAACTCGTGACAACGGTGCACATGTTAACCGTGTTCATCATC } \\
\text { GTTATTTCCGGCTTCACTGGCTTCGCATTATGGTAG }\end{array}$ & Germany & Oct-17 & up_1.2m1 & mid_1.1 \\
\hline
\end{tabular}


Table A1. Cont.

\begin{tabular}{|c|c|c|c|c|c|}
\hline Sample ID & DNA Sequence & Location collected & Date collected & DNA Group & Amino Acid Group \\
\hline up_1.2m2 & $\begin{array}{l}\text { ATGAAGCTGCTACTTTCACATATCGTTATTCTAATATGTTTCAC } \\
\text { CGTCGTGTACAAGATGCTCCCCGGTGGCATGTTCTCGGAAGC } \\
\text { AGACCCGTCGTGGGTTGACTGTCTGTATTTCTCGACGGCAAC } \\
\text { ACACACGACAACGGGCTACGGCGATCTAACGCCAGAAACCC } \\
\text { CGGTGGCAAAACTCGTGACAACGGTGCACATGTTAACCGTG } \\
\text { TTCATCATCGTTATTTCCGGCTTCACTGGCTTCGCATTATGGTAG }\end{array}$ & Germany & Oct-17 & up_1.2m1 & mid_1.1 \\
\hline up_4.1L1 & $\begin{array}{l}\text { ATGAAGCTGCTACTTTCACATATCGTTATTCTAATATGTTTCAC } \\
\text { CGTCGTGTACAAGATGCTCCCCGGTGGCATGTTCTCGGAAGC } \\
\text { AGACCCGTCGTGGGTTGACTGTCTGTATTTCTCGACGGCAAC } \\
\text { ACACACGACAACGGGCTACGGCGATCTAACGCCAGAAACCC } \\
\text { CGGTGGCAAAACTCGTGACAACGGTGCACATGTTAACCGTG } \\
\text { TTCATCATCGTTATTTCCGGCTTCACTGGCTTCGCATTATGGTAG }\end{array}$ & Germany & Oct-17 & up_1.2m1 & mid_1.1 \\
\hline up_4.2 & $\begin{array}{l}\text { ATGAAGCTGCTACTTTCACATATCGTTATTCTAATATGTTTCAC } \\
\text { CGTCGTGTACAAGATGCTCCCCGGTGGCATGTTCTCGGAAG } \\
\text { CAGACCCGTCGTGGGTTGACTGTCTGTATTTCTCGACGGCA } \\
\text { ACACACACGACAACGGGCTACGGCGATCTAACGCCAGAAA } \\
\text { CCCCGGTGGCAAAACTCGTGACAACGGTGCACATGTTAAC } \\
\text { CGTGTTCATCATCGTTATTTCCGGCTTCACTGGCTTCGCATT } \\
\text { ATGGTAG }\end{array}$ & Germany & Oct-17 & up_1.2m1 & mid_1.1 \\
\hline up_7.2 & $\begin{array}{l}\text { ATGAAGCTGCTACTTTCACATATCGTTATTCTAATATGTTTCA } \\
\text { CCGTCGTGTACAAGATGCTCCCCGGTGGCATGTTCTCGGA } \\
\text { AGCAGACCCGTCGTGGGTTGACTGTCTGTATTTCTCGACG } \\
\text { GCAACACACACGACAACGGGCTACGGCGATCTAACGCCA } \\
\text { GAAACCCCGGTGGCAAAACTCGTGACAACGGTGCACAT } \\
\text { GTTAACCGTGTTCATCATCGTTATTTCCGGCTTCACTGG } \\
\text { CTTCGCATTATGGTAG }\end{array}$ & Germany & Oct-17 & up_1.2m1 & mid_1.1 \\
\hline up_8.1 & $\begin{array}{l}\text { ATGAAGCTGCTACTTTCACATATCGTTATTCTAATATGTTTCA } \\
\text { CCGTCGTGTACAAGATGCTCCCCGGTGGCATGTTCTCGGAA } \\
\text { GCAGACCCGTCGTGGGTTGACTGTCTGTATTTCTCGACGGC } \\
\text { AACACACACGACAACGGGCTACGGCGATCTAACGCCAGA } \\
\text { AACCCCGGTGGCAAAACTCGTGACAACGGTGCACATGTT } \\
\text { AACCGTGTTCATCATCGTTATTTCCGGCTTCACTGGCTTCG } \\
\text { CATTATGGTAG }\end{array}$ & Germany & Oct-17 & up_1.2m1 & mid_1.1 \\
\hline
\end{tabular}


Table A1. Cont.

\begin{tabular}{|c|c|c|c|c|c|}
\hline Sample ID & DNA Sequence & Location collected & Date collected & DNA Group & Amino Acid Group \\
\hline mid_3.1s (4) [4] & $\begin{array}{l}\text { ATGAAGCTGCTACTTTCACATATCGTTATTCTAATATGTTTCA } \\
\text { CCGTTATTTACAAGATGCTCCCCGGTGGCATGTTCTCGGAT } \\
\text { GCGGACCCGTCGTGGTTTGACTGTCTGTATTTCTCGACGG } \\
\text { CGACGCATACGACAACAGGCTACGGCGATCTAACGCCTAA } \\
\text { GACGCCGGTGGCAAAACTCGTGACCACAGCGCATATGTTA } \\
\text { ACCGTTTTCGCGATCGTTATTTCCGGTTTCGCTGGCTTCAA } \\
\text { GTTATGGTAG }\end{array}$ & Germany & Oct-17 & mid_3.1s & mid_3.1s \\
\hline mid_14.1 & $\begin{array}{l}\text { ATGAAGCTGCTACTTTCACATATCGTTATTCTAATATGTTTC } \\
\text { ACCGTTATTTACAAGATGCTCCCCGGTGGCATGTTCTCGGA } \\
\text { TGCGGACCCGTCGTGGTTTGACTGTCTGTATTTCTCGACGG } \\
\text { CGACGCATACGACAACAGGCTACGGCGATCTAACGCCTAA } \\
\text { GACGCCGGTGGCAAAACTCGTGACCACAGCGCATATGTTA } \\
\text { ACCGTTTTCGCGATCGTTATTTCCGGTTTCGCTGGCTTCAA } \\
\text { GTTATGGTAG }\end{array}$ & Germany & Oct-17 & mid_3.1s & mid_3.1s \\
\hline mid_14.2 & $\begin{array}{l}\text { ATGAAGCTGCTACTTTCACATATCGTTATTCTAATATGTTTCA } \\
\text { CCGTTATTTACAAGATGCTCCCCGGTGGCATGTTCTCGGATG } \\
\text { CGGACCCGTCGTGGTTTGACTGTCTGTATTTCTCGACGGCGA } \\
\text { CGCATACGACAACAGGCTACGGCGATCTAACGCCTAAGAC } \\
\text { GCCGGTGGCAAAACTCGTGACCACAGCGCATATGTTAACC } \\
\text { GTTTTCGCGATCGTTATTTCCGGTTTCGCTGGCTTCAAGTTA } \\
\text { TGGTAG }\end{array}$ & Germany & Oct-17 & mid_3.1s & mid_3.1s \\
\hline mid_14.3 & $\begin{array}{c}\text { ATGAAGCTGCTACTTTCACATATCGTTATTCTAATATGTTTC } \\
\text { ACCGTTATTTACAAGATGCTCCCCGGTGGCATGTTCTCGGA } \\
\text { TGCGGACCCGTCGTGGTTTGACTGTCTGTATTTCTCGACGG } \\
\text { CGACGCATACGACAACAGGCTACGGCGATCTAACGCCTA } \\
\text { AGACGCCGGTGGCAAAACTCGTGACCACAGCGCATATG } \\
\text { TTAACCGTTTTCGCGATCGTTATTTCCGGTTTCGCTGGC } \\
\text { TTCAAGTTATGGTAG }\end{array}$ & Germany & Oct-17 & mid_3.1s & mid_3.1s \\
\hline mid_6.1 (1) [1] & $\begin{array}{c}\text { ATGAAGCTGCTACTTTCACATATCGTTATTCTAATATGTTTC } \\
\text { ACCGTTATTTACAAGATGCTCCCCGGCGGCATGTTCTCGG } \\
\text { ATGCAGACCCGTCGTGGTTTGACTGTCTGTATTTCTCGAC } \\
\text { GGCGACGCATACGACAACAGGCTACGGCGATCTAACGC } \\
\text { CCAAGTCGCCGGTGGCAAAACTCGTTACCACGGTGCAT } \\
\text { ATGTTAACCGTGTTCGCGATCGTTATTTCCGGGTTCGCTG } \\
\text { GCTTCAAGNTTCCATGGTAG }\end{array}$ & Germany & Oct-17 & mid_6.1 & mid_6.1 \\
\hline
\end{tabular}


Table A1. Cont.

\begin{tabular}{|c|c|c|c|c|c|}
\hline Sample ID & DNA Sequence & Location collected & Date collected & DNA Group & Amino Acid Group \\
\hline mid_6.2 (5) [5] & $\begin{array}{l}\text { ATGAAGCTGCTACTTTCACATATCGTTATTCTAATATGTTTC } \\
\text { ACCGTTATTTACAAGATGCTCCCCGGCGGCATGTTCTCGG } \\
\text { ATGCAGACCCGTCGTGGTTTGACTGTCTGTATTTCTCGAC } \\
\text { GGCGACGCATACGACAACAGGCTACGGCGATCTAACGCC } \\
\text { CAAGTCGCCGGTGGCAAAACTCGTTACCACGGTGCATAT } \\
\text { GTTAACCGTGTTCGCGATCGTTATTTCCGGGTTCGCTGGC } \\
\text { TTCAAGTTTCCATGGTAG }\end{array}$ & Germany & Oct-17 & mid_6.2 & mid_6.2 \\
\hline mid_12.1 & $\begin{array}{c}\text { ATGAAGCTGCTACTTTCACATATCGTTATTCTAATATGTTT } \\
\text { CACCGTTATTTACAAGATGCTCCCCGGCGGCATGTTCTC } \\
\text { GGATGCAGACCCGTCGTGGTTTGACTGTCTGTATTTCTC } \\
\text { GACGGCGACGCATACGACAACAGGCTACGGCGATCTA } \\
\text { ACGCCCAAGTCGCCGGTGGCAAAACTCGTTACCACG } \\
\text { GTGCATATGTTAACCGTGTTCGCGATCGTTATTTCCGG } \\
\text { GTTCGCTGGCTTCAAGTTTCCATGGTAG }\end{array}$ & Germany & Oct-17 & mid_6.2 & mid_6.2 \\
\hline mid_12.3L & $\begin{array}{c}\text { ATGAAGCTGCTACTTTCACATATCGTTATTCTAATAT } \\
\text { GTTTCACCGTTATTTACAAGATGCTCCCCGGCGGCA } \\
\text { TGTTCTCGGATGCAGACCCGTCGTGGTTTGACTGT } \\
\text { CTGTATTTCTCGACGGCGACGCATACGACAACAGG } \\
\text { CTACGGCGATCTAACGCCCAAGTCGCCGGTGGCAA } \\
\text { AACTCGTTACCACGGTGCATATGTTAACCGTGTTCG } \\
\text { CGATCGTTATTTCCGGGTTCGCTGGCTTCAAGTTT } \\
\text { CCATGGTAG }\end{array}$ & Germany & Oct-17 & mid_6.2 & mid_6.2 \\
\hline mid_12.3s & $\begin{array}{c}\text { ATGAAGCTGCTACTTTCACATATCGTTATTCTAATATG } \\
\text { TTTCACCGTTATTTACAAGATGCTCCCCGGCGGCAT } \\
\text { GTTCTCGGATGCAGACCCGTCGTGGTTTGACTGTCT } \\
\text { GTATTTCTCGACGGCGACGCATACGACAACAGGCT } \\
\text { ACGGCGATCTAACGCCCAAGTCGCCGGTGGCAAA } \\
\text { ACTCGTTACCACGGTGCATATGTTAACCGTGTTCG } \\
\text { CGATCGTTATTTCCGGGTTCGCTGGCTTCAAGTTT } \\
\text { CCATGGTAG }\end{array}$ & Germany & Oct-17 & mid_6.2 & mid_6.2 \\
\hline
\end{tabular}


Table A1. Cont.

\begin{tabular}{|c|c|c|c|c|c|}
\hline Sample ID & DNA Sequence & Location collected & Date collected & DNA Group & Amino Acid Group \\
\hline mid_8.1 & $\begin{array}{c}\text { ATGAAGCTGCTACTTTCACATATCGTTATTCTAATAT } \\
\text { GTTTCACCGTTATTTACAAGATGCTCCCCGGCGGC } \\
\text { ATGTTCTCGGATGCAGACCCGTCGTGGTTTGACTG } \\
\text { TCTGTATTTCTCGACGGCGACGCATACGACAACA } \\
\text { GGCTACGGCGATCTAACGCCCAAGTCGCCGGTGG } \\
\text { CAAAACTCGTTACCACGGTGCATATGTTAACCGTG } \\
\text { TTCGCGATCGTTATTTCCGGGTTCGCTGGCTTCAA } \\
\text { GTTTCCATGGTAG }\end{array}$ & Germany & Oct-17 & mid_6.2 & mid_6.2 \\
\hline mid_9.1 (1) [1] & $\begin{array}{c}\text { ATGAAGCTGCTACTTTCACATATCGTTATTCTAAT } \\
\text { ATGTTTCACCGTTATTTACAAGATGCTCCCCGGTG } \\
\text { GCATGTTCTCGGATGCGGACCCGTCGTGGTTTGA } \\
\text { CTGTCTGTATTTCTCGACGGCGACGCATACGACA } \\
\text { ACAGGCTACGGCGATCTAACGCCTAAGTCGCCG } \\
\text { GTGGCAAAACTCGTGACCACGGTGCATATGTTA } \\
\text { ACCGTTTTCGCGATCGTTATTTCCGGGTTCGCTG } \\
\text { GCTTCAAGTTATGGTAG }\end{array}$ & Germany & Oct-17 & mid_9.1 & mid_9.1 \\
\hline MN08101 (1) [1] & $\begin{array}{c}\text { ATGCTGCTTCTCCTGATACACATTGCCATATTGACATTC } \\
\text { TTTACGGTCGTGTACAAGATGCTCCCCGACGGCGTG } \\
\text { TTCTCGAACGGGGACCCGTCGTGGGTAGACTGCTTAT } \\
\text { ACTTTTCCGCGTCCACTCACACCACCGTGGGATACGG } \\
\text { GGACCTCACCCCCAAATCACCCGTGGCAAAACTCAC } \\
\text { GGCAACGGCCCATATGATGATCGTGTTCGCGATTGTAG } \\
\text { TGTCTAGCTTCACGTTCCCGTGGTAG }\end{array}$ & Minnesota & 2008 & MN08101 & MN08101 \\
\hline NEJV2 (2) [6] & $\begin{array}{c}\text { ATGTTGCTGCTTATCATACATATCATCATTCTGATAGTGT } \\
\text { TCACTACCATCTACAAGATGCTCCCCGGTGGTATGTTC } \\
\text { TCGAACACGGACCCGACTTGGGTTGATTGCCTGTACT } \\
\text { TTTCGGCATCGACGCACACCACTGTGGGGTACGGAGA } \\
\text { TCTCACGCCCAAATCACCCGTGGCAAAACTCACGGC } \\
\text { AACGGCACACATGTTGATCGTATTCGCGATCGTCATTT } \\
\text { CCGGCTTCACGTTTTCGTGGTAG }\end{array}$ & $\begin{array}{l}\text { Rowe Bird Sanctuary; } \\
\text { Gibbon, Nebraska }\end{array}$ & 2008 & NEJV2 & NEJV2 \\
\hline Dismal_NE_s3 & $\begin{array}{l}\text { ATGTTGCTGCTTATCATACATATCATCATTCTGATAGTG } \\
\text { TTCACTACCATCTACAAGATGCTCCCCGGTGGTATGTTCTCGAA } \\
\text { CACGGACCCGACTTGGGTTGATTGCCTGTACTTTTCGGCATCGA } \\
\text { CGCACACCACTGTGGGGTACGGAGATCTCACGCCCAAATCAC } \\
\text { CCGTGGCAAAACTCACGGCAACGGCACACATGTTGATCGTAT } \\
\text { TCGCGATCGTCATTTCCGGCTTCACGTTTTCGTGGTAG }\end{array}$ & $\begin{array}{l}\text { Dismal River, } \\
\text { Nebraska }\end{array}$ & $5 / 26 / 2017$ & NEJV2 & NEJV2 \\
\hline
\end{tabular}


Table A1. Cont.

\begin{tabular}{|c|c|c|c|c|c|}
\hline Sample ID & DNA Sequence & Location collected & Date collected & DNA Group & Amino Acid Group \\
\hline NEJV3 (1) & $\begin{array}{l}\text { ATGTTGCTGCTTATCATACATATCATCATTCTGATAGTGTTCACT } \\
\text { ACCATCTACAAGATGCTCCCCGGCGGCATGTTCTCGAACACA } \\
\text { GACCCGACTTGGGTTGATTGCCTGTACTTTTCGGCATCGACG } \\
\text { CACACCACTGTGGGGTACGGAGATCTCACGCCCAAATCACC } \\
\text { CGTGGCAAAACTCACGGCAACGGCACACATGTTGATCGTAT } \\
\text { TCGCGATCGTCATTTCCGGCTTCACGTTTTCGTGGTAG }\end{array}$ & $\begin{array}{l}\text { Gudmundsen } \\
\text { Ranch, NE }\end{array}$ & 2008 & NEJV3 & NEJV2 \\
\hline MO3 (3) & $\begin{array}{l}\text { ATGTTGCTGCTTATCATACATATCATCATTCTGATAGTGTTCAC } \\
\text { TACCATCTACAAGATGCTCCCCGGCGGCATGTTCTCGAACA } \\
\text { CAGACCCTACTTGGGTTGATTGCCTGTACTTTTCGGCATCGA } \\
\text { CGCACACCACCGTGGGGTACGGAGATCTCACGCCCAAATC } \\
\text { ACCCGTGGCAAAACTCACGGCAACGGCACACATGTTGATC } \\
\text { GTATTCGCGATCGTCATTTCCGGCTTCACGTTTTCGTGGTAG }\end{array}$ & $\begin{array}{l}\text { Lake Lotawana, } \\
\text { Missouri }\end{array}$ & $5 / 31 / 2017$ & MO3 & NEJV2 \\
\hline $\mathrm{NY} 2 \mathrm{~m}$ & $\begin{array}{l}\text { ATGTTGCTGCTTATCATACATATCATCATTCTGATAGTGTTCA } \\
\text { CTACCATCTACAAGATGCTCCCCGGCGGCATGTTCTCGAA } \\
\text { CACAGACCCTACTTGGGTTGATTGCCTGTACTTTTCGGCA } \\
\text { TCGACGCACACCACCGTGGGGTACGGAGATCTCACGCC } \\
\text { CAAATCACCCGTGGCAAAACTCACGGCAACGGCACACA } \\
\text { TGTTGATCGTATTCGCGATCGTCATTTCCGGCTTCACGTT } \\
\text { TTCGTGGTAG }\end{array}$ & $\begin{array}{c}\text { Moodna Creek; } \\
\text { Washingtonville, New } \\
\text { York }\end{array}$ & $6 / 21 / 2017$ & MO3 & NEJV2 \\
\hline Verbena_VA_s3 & $\begin{array}{c}\text { ATGTTGCTGCTTATCATACATATCATCATTCTGATAGTGT } \\
\text { TCACTACCATCTACAAGATGCTCCCCGGCGGCATGTTC } \\
\text { TCGAACACAGACCCTACTTGGGTTGATTGCCTGTACTTT } \\
\text { TCGGCATCGACGCACACCACCGTGGGGTACGGAGATCT } \\
\text { CACGCCCAAATCACCCGTGGCAAAACTCACGGCAACG } \\
\text { GCACACATGTTGATCGTATTCGCGATCGTCATTTCCGGC } \\
\text { TTCACGTTTTCGTGGTAG }\end{array}$ & $\begin{array}{l}\text { South fork of the } \\
\text { Shenandoah River; } \\
\text { Verbena, Virginia }\end{array}$ & 7/1/2017 & MO3 & NEJV2 \\
\hline NTS1 (1) [1] & $\begin{array}{c}\text { ATGTTGCTGCTTCTCATACATCTCAGCATTTTGGTAATTTT } \\
\text { CACTGCCATCTACAAGATGCTGCCCGGCGGCATGTTCTC } \\
\text { AAACACAGACCCGACTTGGGTCGATTGCCTGTACTTTTC } \\
\text { GGCATCAACGCACACCACCGTGGGGTACGGGGACCTC } \\
\text { ACGCCAAAATCACCCGTGGCAAAACTCACGGCAACGG } \\
\text { CACACATGCTGATCGTATTCGCGATCGTCATTTCTGGCTT } \\
\text { CACGTTCCCGTGGTAG }\end{array}$ & $\begin{array}{l}\text { Next to Smith Lake, } \\
\text { Western Nebraska }\end{array}$ & 2008 & NTS1 & NTS1 \\
\hline
\end{tabular}


Table A1. Cont.

\begin{tabular}{|c|c|c|c|c|c|}
\hline Sample ID & DNA Sequence & Location collected & Date collected & DNA Group & Amino Acid Group \\
\hline NY-2 (1) [4] & $\begin{array}{c}\text { ATGTTGCTGCTTCTCATACATCTCAGCATTTTGGTAATTTTC } \\
\text { ACTACCATCTACAAGATGCTTCCCGGCGGCATGTTCTCGAA } \\
\text { CACGGACCCATCCTGGGTCGATTGCCTGTACTTTTCGGCAT } \\
\text { CAACACACACCACCGTGGGGTACGGGGACCTCACGCCAA } \\
\text { AATCACCCGTGGCAAAACTCACGGCAACGGCACACATGC } \\
\text { TGATCGTTTTCGCGATCGTCATTTCTGGCTTCACGTT } \\
\text { CCCGTGGTAG }\end{array}$ & $\begin{array}{c}\text { Moodna Creek; } \\
\text { Washingtonville, New } \\
\text { York }\end{array}$ & $6 / 21 / 2017$ & NY-2 & NY-2 \\
\hline $\begin{array}{c}\text { Somers_MT_L3 } \\
\text { (1) }\end{array}$ & $\begin{array}{c}\text { ATGTTGCTGCTTCTCATACATCTCAGCATTTTGGTAATTTTC } \\
\text { ACTACCATCTACAAGATGCTCCCCGGCGGCATGTTCTCGA } \\
\text { ACACGGACCCATCCTGGGTCGATTGCCTGTACTTTTCGGC } \\
\text { ATCAACGCACACCACCGTGGGGTACGGGGACCTCACGCC } \\
\text { AAAATCACCCGTGGCAAAACTCACGGCAACGGCACACA } \\
\text { TGCTGATCGTATTCGCGATCGTCATTTCTGGCTTCACGT } \\
\text { TCCCGTGGTAA }\end{array}$ & $\begin{array}{c}\text { Flathead Lake; Somers, } \\
\text { Montana }\end{array}$ & $6 / 27 / 2017$ & Somers_MT_L3 & NY-2 \\
\hline $\begin{array}{c}\text { Verbena_VA_L4 } \\
\text { (2) }\end{array}$ & $\begin{array}{c}\text { ATGTTGCTGCTTCTCATACATCTCAGCATTTTGGTAATTTT } \\
\text { CACTACCATCTACAAGATGCTTCCCGGCGGCATGTTCTCG } \\
\text { AACACGGACCCATCCTGGGTCGATTGCCTGTACTTTTCG } \\
\text { GCATCAACGCACACCACCGTGGGGTACGGGGACCTCAC } \\
\text { GCCAAAATCACCCGTGGCAAAACTCACGGCAACGGCA } \\
\text { CACATGCTGATCGTTTTCGCGATCGTCATTTCTGGCTTC } \\
\text { ACGTTCCCGTGGTAG }\end{array}$ & $\begin{array}{l}\text { South fork of the } \\
\text { Shenandoah River; } \\
\text { Verbena, Virginia }\end{array}$ & $7 / 1 / 2017$ & Verbena_VA_L4 & NY-2 \\
\hline Verbena_VA_m1 & $\begin{array}{c}\text { ATGTTGCTGCTTCTCATACATCTCAGCATTTTGGTAATTTT } \\
\text { CACTACCATCTACAAGATGCTTCCCGGCGGCATGTTCTC } \\
\text { GAACACGGACCCATCCTGGGTCGATTGCCTGTACTTTTC } \\
\text { GGCATCAACGCACACCACCGTGGGGTACGGGGACCTC } \\
\text { ACGCCAAAATCACCCGTGGCAAAACTCACGGCAACGG } \\
\text { CACACATGCTGATCGTTTTCGCGATCGTCATTTCTGGCT } \\
\text { TCACGTTCCCGTGGTAG } \\
\end{array}$ & $\begin{array}{l}\text { South fork of the } \\
\text { Shenandoah River; } \\
\text { Verbena, Virginia }\end{array}$ & $7 / 1 / 2017$ & Verbena_VA_L4 & NY-2 \\
\hline $\begin{array}{c}\text { Smith_L_La (3) } \\
{[3]}\end{array}$ & $\begin{array}{c}\text { ATGTTGCTGCTCCTTATCCACATGTGTATTTTGACATTCTT } \\
\text { CACAGTTGTTTACAAGATGCTCCCTGGCGGCATGTTCTC } \\
\text { TAACGCGGACCCGTCGTGGGTAGACTGCTTATACTTTGC } \\
\text { CGCGTCGACTCACACCACGGTGGGGTACGGGGACCTCA } \\
\text { CCCCCAAATCGCCAGTGGCAAAGCTCACGGCGACGGC } \\
\text { CCACATGTTGATCGTGTTCGCGATCATTATATCTAGCTTC } \\
\text { ACACTCCCATGGTAG }\end{array}$ & $\begin{array}{c}\text { Smith Lake, Western } \\
\text { Nebraska }\end{array}$ & $10 / 22 / 2017$ & Smith_L_La & Smith_L_La \\
\hline
\end{tabular}


Table A1. Cont.

\begin{tabular}{|c|c|c|c|c|c|}
\hline Sample ID & DNA Sequence & Location collected & Date collected & DNA Group & Amino Acid Group \\
\hline $\begin{array}{l}\text { Smith-Lake_ } \\
\text { Medium-Plaque }\end{array}$ & $\begin{array}{c}\text { ATGTTGCTGCTCCTTATCCACATGTGTATTTTGACATTCT } \\
\text { TCACAGTTGTTTACAAGATGCTCCCTGGCGGCATGTTC } \\
\text { TCTAACGCGGACCCGTCGTGGGTAGACTGCTTATACTT } \\
\text { TGCCGCGTCGACTCACACCACGGTGGGGTACGGGGAC } \\
\text { CTCACCCCCAAATCGCCAGTGGCAAAGCTCACGGCGA } \\
\text { CGGCCCACATGTTGATCGTGTTCGCGATCATTATATCTA } \\
\text { GCTTCACACTCCCATGGTAG }\end{array}$ & $\begin{array}{c}\text { Smith Lake, Western } \\
\text { Nebraska }\end{array}$ & 2018 & Smith_L_La & Smith_L_La \\
\hline $\begin{array}{l}\text { Island-Lake_ } \\
\text { Large-Plaque }\end{array}$ & $\begin{array}{l}\text { ATGTTGCTGCTCCTTATCCACATGTGTATTTTGACATTCTTC } \\
\text { ACAGTTGTTTACAAGATGCTCCCTGGCGGCATGTTCTCTA } \\
\text { ACGCGGACCCGTCGTGGGTAGACTGCTTATACTTTGCCGC } \\
\text { GTCGACTCACACCACGGTGGGGTACGGGGACCTCACCC } \\
\text { CCAAATCGCCAGTGGCAAAGCTCACGGCGACGGCCCAC } \\
\text { ATGTTGATCGTGTTCGCGATCATTATATCTAGCTTCACACT } \\
\text { CCCATGGTAG }\end{array}$ & $\begin{array}{c}\text { Island Lake, Western } \\
\text { Nebraska }\end{array}$ & 2018 & Smith_L_La & Smith_L_La \\
\hline SPRLa (1) [1] & $\begin{array}{l}\text { ATGTTGCTGCTCCTTATACACATCGGTATTTTGGTATTTTTC } \\
\text { ACTATCGTGTACAAGATGCTCCCCGGCGGCATGTTCTCGA } \\
\text { ACACAGACCCTACTTGGGTCGATTGCCTGTACTTTTCGGC } \\
\text { ATCGACGCACACCACCGTGGGGTACGGAGATCTCACGCC } \\
\text { CAAATCACCCGTGGCAAAACTCACGGCAACGGCACACAT } \\
\text { GTTGATCGTATTCGCGATCGTCATTTCCGGCTTCACGTTTC } \\
\text { CGTGGTAG }\end{array}$ & $\begin{array}{l}\text { South Platte River; Big } \\
\text { Spring, Nebraska }\end{array}$ & $10 / 23 / 2017$ & SPRLa & SPRLa \\
\hline TN603 (1) [1] & $\begin{array}{l}\text { ATGTTGCTGCTTCTCATACACCTCTGTATTTTGATAATTTTTA } \\
\text { CTACAATATACAAGATGTTGCCCGGAGGCATGTTCTCGAAC } \\
\text { ACGGACCCGTCATGGATAGATTGCCTGTACTTCTCGGCATC } \\
\text { AACGCACACCACCGTGGGGTACGGGGATCTCACGCCCAAA } \\
\text { TCGCCCGTGGCAAAACTCACAGCAACGGCACACATGCTG } \\
\text { ATCGTATTCGCGATCGTAATAACTGGCTTCACATTCCCG } \\
\text { TGGTAA }\end{array}$ & Tennessee & 2006 & TN603 & TN603 \\
\hline up_3.1 (4) [4] & $\begin{array}{c}\text { ATGAAGCTGCTACTTTCACATATCGTTATTCTAATATGTTT } \\
\text { CACCGTTATTTACAAGATGCTCCCCGGTGGCATGTTCTCG } \\
\text { GATGCGGACCCGTCGTGGTTTGACTGTCTGTATTTCTCGA } \\
\text { CGGCGACGCATACGACAACAGGCTACGGCGATCTAACG } \\
\text { CCTAAGTCGCCGGTGGCAAAACTCGTGACCACAGCGCA } \\
\text { TATGTTAACCGTTTTCGCGATCGTTATTTCCGGTTTCGCTG } \\
\text { GCTTCAAGTTATGGTAG }\end{array}$ & Germany & Oct-17 & up_3.1 & up_3.1 \\
\hline
\end{tabular}


Table A1. Cont.

\begin{tabular}{|c|c|c|c|c|c|}
\hline Sample ID & DNA Sequence & Location collected & Date collected & DNA Group & Amino Acid Group \\
\hline up_5.2L & $\begin{array}{c}\text { ATGAAGCTGCTACTTTCACATATCGTTATTCTAATATGTTTC } \\
\text { ACCGTTATTTACAAGATGCTCCCCGGTGGCATGTTCTCGG } \\
\text { ATGCGGACCCGTCGTGGTTTGACTGTCTGTATTTCTCGAC } \\
\text { GGCGACGCATACGACAACAGGCTACGGCGATCTAACG } \\
\text { CCTAAGTCGCCGGTGGCAAAACTCGTGACCACAGCGC } \\
\text { ATATGTTAACCGTTTTCGCGATCGTTATTTCCGGTTTCGC } \\
\text { TGGCTTCAAGTTATGGTAG }\end{array}$ & Germany & Oct-17 & up_3.1 & up_3.1 \\
\hline up_5.3m & $\begin{array}{c}\text { ATGAAGCTGCTACTTTCACATATCGTTATTCTAATATGTTT } \\
\text { CACCGTTATTTACAAGATGCTCCCCGGTGGCATGTTCTC } \\
\text { GGATGCGGACCCGTCGTGGTTTGACTGTCTGTATTTCTC } \\
\text { GACGGCGACGCATACGACAACAGGCTACGGCGATCTA } \\
\text { ACGCCTAAGTCGCCGGTGGCAAAACTCGTGACCACAG } \\
\text { CGCATATGTTAACCGTTTTCGCGATCGTTATTTCCGGTT } \\
\text { TCGCTGGCTTCAAGTTATGGTAG }\end{array}$ & Germany & Oct-17 & up_3.1 & up_3.1 \\
\hline up_5.3s2 & $\begin{array}{c}\text { ATGAAGCTGCTACTTTCACATATCGTTATTCTAATATGTTT } \\
\text { CACCGTTATTTACAAGATGCTCCCCGGTGGCATGTTCTC } \\
\text { GGATGCGGACCCGTCGTGGTTTGACTGTCTGTATTTCTC } \\
\text { GACGGCGACGCATACGACAACAGGCTACGGCGATCTAA } \\
\text { CGCCTAAGTCGCCGGTGGCAAAACTCGTGACCACAGC } \\
\text { GCATATGTTAACCGTTTTCGCGATCGTTATTTCCGGTTTC } \\
\text { GCTGGCTTCAAGTTATGGTAG }\end{array}$ & Germany & Oct-17 & up_3.1 & up_3.1 \\
\hline
\end{tabular}

Sequences submitted to the GenBank. The accession numbers for the kcv sequences in GenBank are MT560092-MT560194. 


\section{Appendix B}

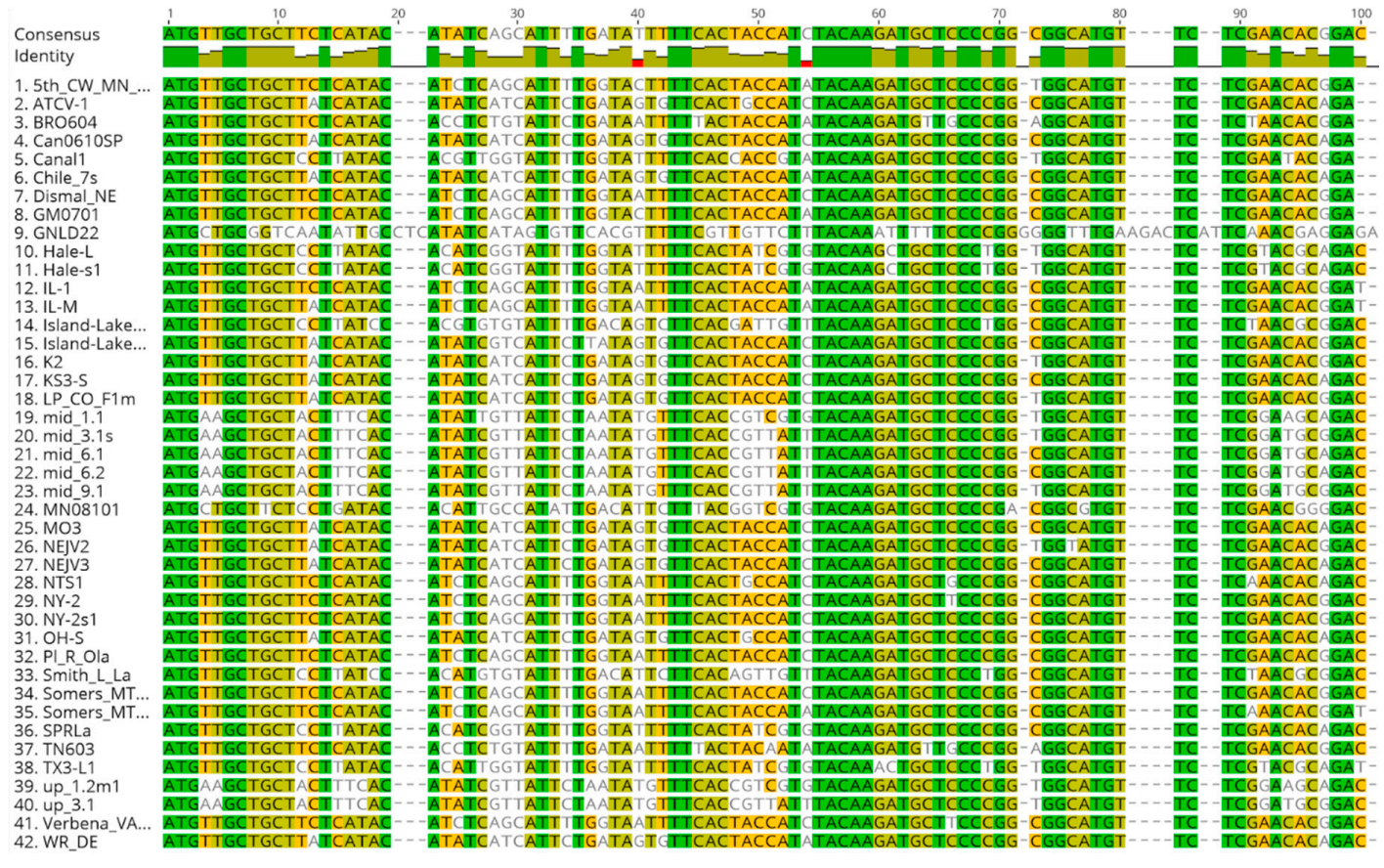

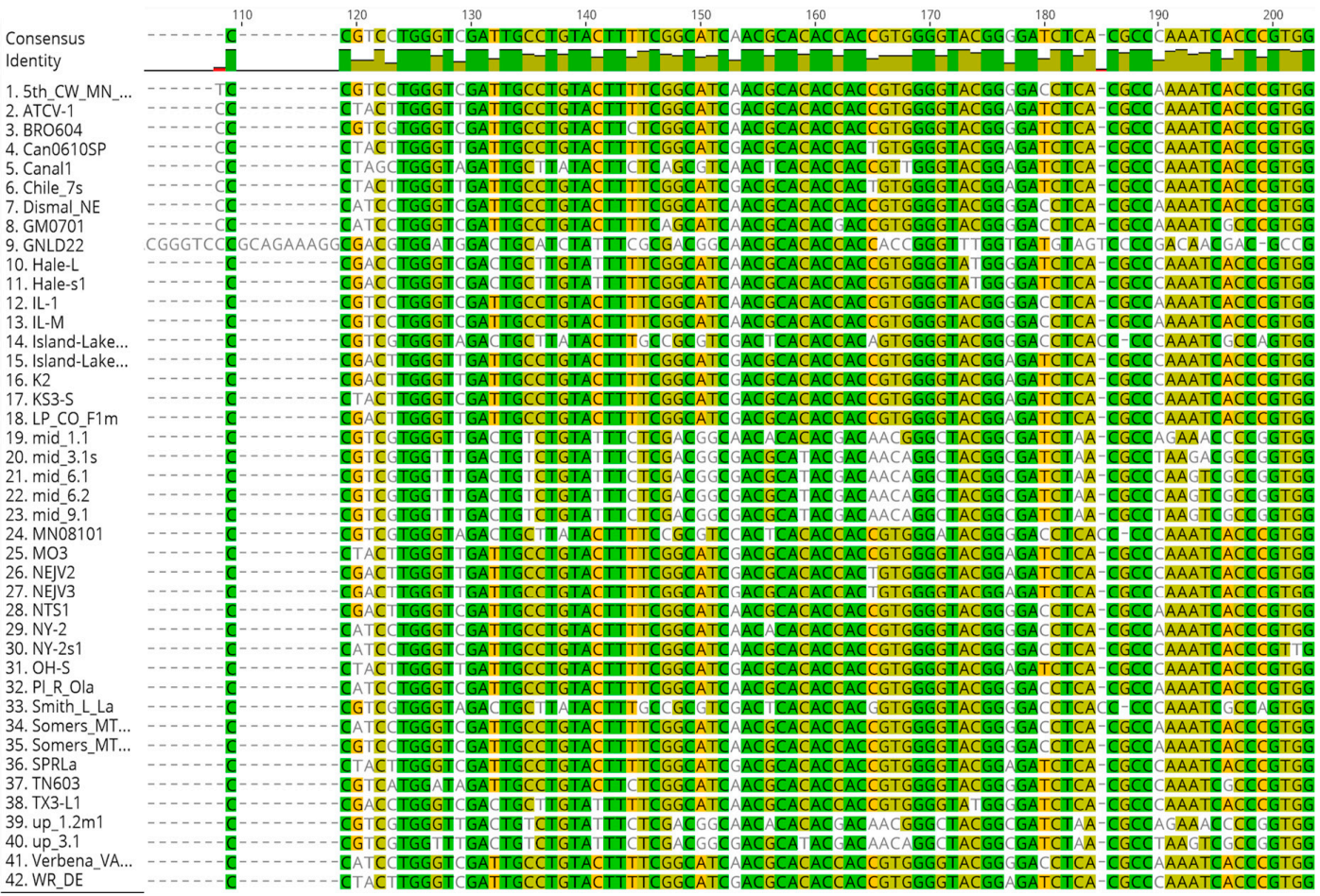

Figure A1. Cont. 


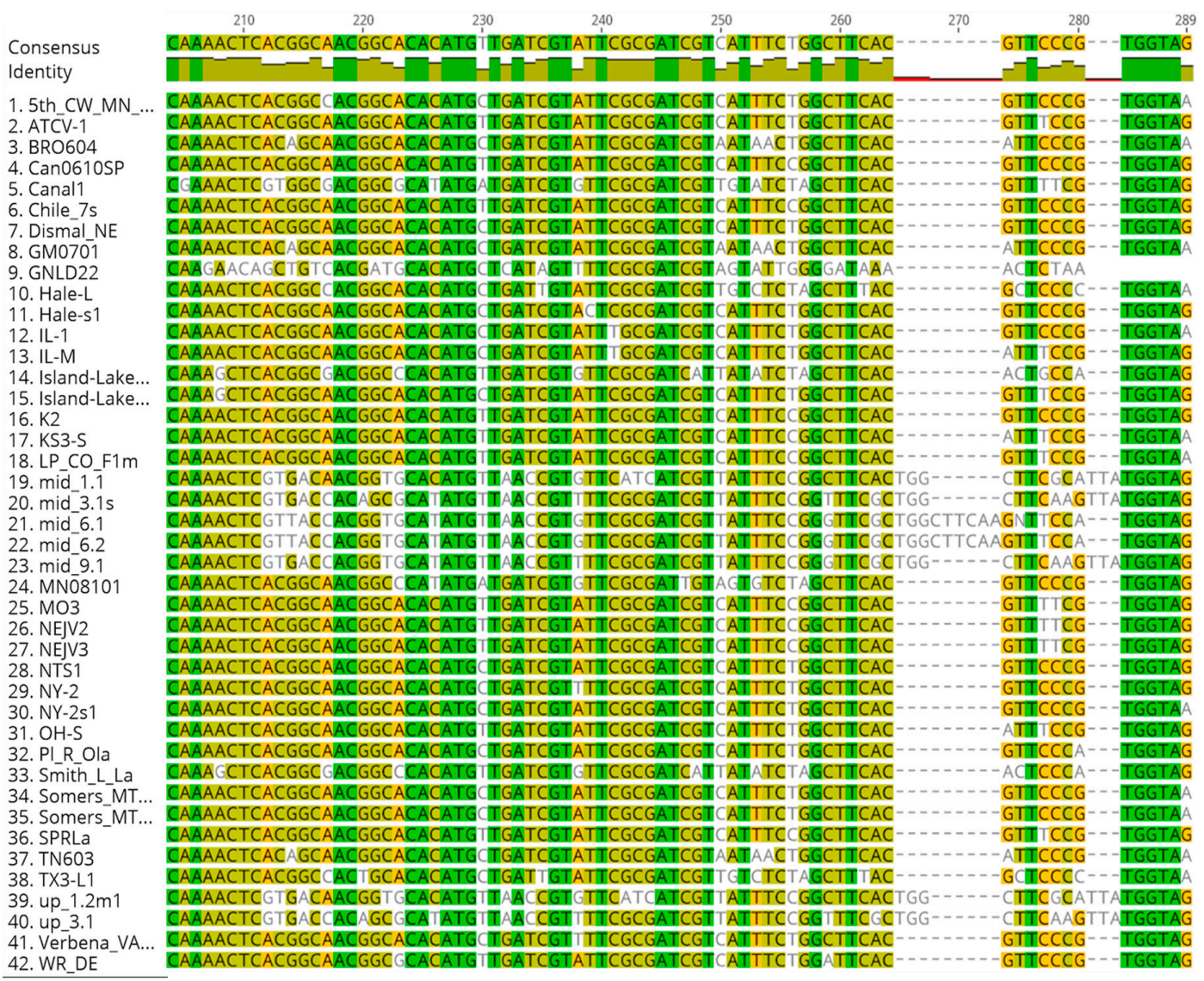

Figure A1. DNA sequence alignment of unique SAG chlorovirus $k c v$ genes. DNA sequences were aligned using the Geneious Alignment algorithm from Geneious 11.0.5 software. Green color indicates identical nucletides among all sequenced strains. Different shades from olive to orange denote different degrees of conservation with olive color being the most conserved.

\section{References}

1. Van Etten, J.L.; Agarkova, I.V.; Dunigan, D.D. Chloroviruses. Viruses 2020, 12, 20. [CrossRef]

2. Cherrier, M.V.; Kostyuchenko, V.A.; Xiao, C.; Bowman, V.D.; Battisti, A.J.; Yan, X.; Chipman, P.R.; Baker, T.S.; Van Etten, J.L.; Rossmann, M.G. An icosahedral algal virus has a complex unique vertex decorated by a spike. Proc. Natl. Acad. Sci. USA 2009, 106, 11085-11089. [CrossRef]

3. Plugge, B.; Gazzarrini, S.; Nelson, M.; Cerana, R.; Van Etten, J.L.; Derst, C.; DiFrancesco, D.; Moroni, A.; Thiel, G. A potassium channel protein encoded by chlorella virus PBCV-1. Science 2000, 287, 1641-1644. [CrossRef]

4. Milrot, E.; Shimoni, E.; Dadosh, T.; Rechav, K.; Unger, T.; Van Etten, J.L.; Minsky, A. Structural studies demonstrating a bacteriophage-like replication cycle of the eukaryote-infecting Paramecium bursaria chlorella virus-1. PLoS Pathog. 2017, 13, e1006562. [CrossRef] [PubMed]

5. Romani, G.; Piotrowski, A.; Hillmer, S.; Gurnon, J.; Van Etten, J.L.; Moroni, A.; Thiel, G.; Hertel, B. A virus-encoded potassium ion channel is a structural protein in the chlorovirus Paramecium bursaria chlorella virus 1 virion. J. Gen. Virol. 2013, 94, 2549-2556. [CrossRef] [PubMed]

6. Frohns, F.; Käsmann, A.; Kramer, D.; Schäfer, B.; Mehmel, M.; Kang, M.; Van Etten, J.L.; Gazzarrini, S.; Moroni, A.; Thiel, G. Potassium ion channels of chlorella viruses cause rapid depolarization of host cells during infection. J. Virol. 2006, 80, 2437-2444. [CrossRef] [PubMed]

7. Lee, S.-W.; Lee, E.-H.; Thiel, G.; Van Etten, J.L.; Saraf, R.F. Noninvasive measurement of electrical events associated with a single chlorovirus infection of a microalgal cell. ACS Nano 2016, 10, 5123-5130. [CrossRef]

8. Neupärtl, M.; Meyer, C.; Woll, I.; Frohns, F.; Kang, M.; Van Etten, J.L.; Kramer, D.; Hertel, B.; Moroni, A.; Thiel, G. Chlorella viruses evoke a rapid release of $\mathrm{K}^{+}$from host cells during early phase of infection. Virology 2008, 372, 340-348. [CrossRef] 
9. Thiel, G.; Moroni, A.; Dunigan, D.; Van Etten, J.L. Initial events associated with virus PBCV-1 infection of Chlorella NC64A. Prog. Bot. 2010, 71, 169-183.

10. Agarkova, I.; Dunigan, D.; Gurnon, J.; Greiner, T.; Barres, J.; Thiel, G.; Van Etten, J. Chlorovirus-mediated membrane depolarization of Chlorella alters secondary active transport of solutes. J. Virol. 2008, 82, 12181-12190. [CrossRef]

11. Chase, T.E.; Nelson, J.A.; Burbank, D.E.; Van Etten, J.L. Mutual exclusion occurs in a Chlorella-like green alga inoculated with two viruses. J. Gen. Virol. 1989, 70, 1829-1836. [CrossRef] [PubMed]

12. Gazzarrini, S.; Kang, M.; Abenavoli, A.; Romani, G.; Olivari, C.; Gaslini, D.; Ferrara, G.; Van Etten, J.L.; Kreim, M.; Kast, S.M.; et al. Chlorella virus ATCV-1 encodes a functional potassium channel of 82 amino acids. Biochem. J. 2009, 420, 295-303. [CrossRef] [PubMed]

13. Van Etten, J.L.; Burbank, D.E.; Kuczmarski, D.; Meints, R.H. Virus infection of culturable chlorella-like algae and development of a plaque assay. Science 1983, 219, 994-996. [CrossRef] [PubMed]

14. Jeanniard, A.; Dunigan, D.D.; Gurnon, J.R.; Agarkova, I.V.; Kang, M.; Vitek, J.; Duncan, G.; McClung, O.W.; Larsen, M.; Claverie, J.-M.; et al. Towards defining the chloroviruses: A genomic journey through a genus of large DNA viruses. BMC Genom. 2013, 14, 1-14. [CrossRef] [PubMed]

15. Winterstein, L.M.; Kukovetz, K.; Rauh, O.; Turman, D.L.; Braun, C.; Moroni, A.; Schroeder, I.; Thiel, G. Reconstitution and functional characterization of ion channels from nanodiscs in lipid bilayers. J. Gen. Physiol. 2018, 150, 637-646. [CrossRef]

16. Braun, C.J.; Lachnit, C.; Becker, P.; Henkes, L.M.; Arrigoni, C.; Kast, S.M.; Moroni, A.; Thiel, G.; Schroeder, I. Viral potassium channels as a robust model sysem for studies of membrane-protein interaction. Biochim. Biophys. Acta 2014, 1838, 1096-1103. [CrossRef]

17. Gazzarrini, S.; Kang, M.; Epimashko, S.; Van Etten, J.L.; Dainty, J.; Thiel, G.; Moroni, A. Chlorella virus MT325 encodes water and potassium channels that interact synergistically. Proc. Natl. Acad. Sci. USA 2006, 103, 5355-5360. [CrossRef] [PubMed]

18. Rauh, O.; Urban, M.; Henkes, L.M.; Winterstein, T.; Greiner, T.; Van Etten, J.L.; Moroni, A.; Kast, S.M.; Thiel, G.; Schroeder, I. Sequence specific distortions of a transmembrane helix generate a gate in a $\mathrm{K}^{+}$channel with a long lived closed state. J. Am. Chem. Soc. 2017, 139, 7494-7503. [CrossRef]

19. Mehmel, M.; Rothermel, M.; Meckel, T.; Van Etten, J.L.; Moroni, A.; Thiel, G. Possible function for virus encoded $\mathrm{K}^{+}$channel Kcv in the replication of chlorella virus PBCV-1. FEBS Lett. 2003, 552, 7-11. [CrossRef]

20. Kang, M.; Moroni, A.; Gazzarrini, S.; DiFrancesco, D.; Thiel, G.; Severino, M.; Van Etten, J.L. Small potassium ion channel proteins encoded by chlorella viruses. Proc. Natl. Acad. Sci. USA 2004, 101, 5318-5324. [CrossRef]

21. Gazzarrini, S.; Kang, M.; Van Etten, J.L.; Tayefeh, S.; Kast, S.M.; DiFrancesco, D.; Thiel, G.; Moroni, A. Long distance interactions within the potassium channel pore are revealed by molecular diversity of viral proteins. J. Biol. Chem. 2004, 279, 28443-28449. [CrossRef] [PubMed]

22. Siotto, F.; Martin, C.; Rauh, O.; Van Etten, J.L.; Schoeder, I.; Moroni, A.; Thiel, G. Viruses infecting marine picoplancton encode function potassium ion channels. Virology 2014, 466, 103-111. [CrossRef] [PubMed]

(C) 2020 by the authors. Licensee MDPI, Basel, Switzerland. This article is an open access article distributed under the terms and conditions of the Creative Commons Attribution (CC BY) license (http://creativecommons.org/licenses/by/4.0/). 